\title{
Jet shapes in dijet events at the LHC in SCET
}

\author{
Andrew Hornig, ${ }^{a}$ Yiannis Makris ${ }^{b}$ and Thomas Mehen ${ }^{b}$ \\ ${ }^{a}$ Theoretical Division T-2, Los Alamos National Laboratory, \\ Los Alamos, NM 87545, U.S.A. \\ ${ }^{b}$ Department of Physics, Duke University, \\ Durham, NC 27708, U.S.A. \\ E-mail: ahornig@lanl.gov, yiannis.makris@duke.edu, mehen@phy.duke.edu
}

ABStRaCT: We consider the class of jet shapes known as angularities in dijet production at hadron colliders. These angularities are modified from the original definitions in $e^{+} e^{-}$ collisions to be boost invariant along the beam axis. These shapes apply to the constituents of jets defined with respect to either $k_{T}$-type (anti- $k_{T}, C / A$, and $k_{T}$ ) algorithms and conetype algorithms. We present an SCET factorization formula and calculate the ingredients needed to achieve next-to-leading-log (NLL) accuracy in kinematic regions where nonglobal logarithms are not large. The factorization formula involves previously unstudied "unmeasured beam functions," which are present for finite rapidity cuts around the beams. We derive relations between the jet functions and the shape-dependent part of the soft function that appear in the factorized cross section and those previously calculated for $e^{+} e^{-}$collisions, and present the calculation of the non-trivial, color-connected part of the soft-function to $\mathcal{O}\left(\alpha_{s}\right)$. This latter part of the soft function is universal in the sense that it applies to any experimental setup with an out-of-jet $p_{T}$ veto and rapidity cuts together with two identified jets and it is independent of the choice of jet (sub-)structure measurement. In addition, we implement the recently introduced soft-collinear refactorization to resum logarithms of the jet size, valid in the region of non-enhanced non-global logarithm effects. While our results are valid for all $2 \rightarrow 2$ channels, we compute explicitly for the $q q^{\prime} \rightarrow$ $q q^{\prime}$ channel the color-flow matrices and plot the NLL resummed differential dijet cross section as an explicit example, which shows that the normalization and scale uncertainty is reduced when the soft function is refactorized. For this channel, we also plot the jet size $R$ dependence, the $p_{T}^{\text {cut }}$ dependence, and the dependence on the angularity parameter $a$.

KeYWORDS: Jets, QCD Phenomenology

ARXIV EPRINT: 1601.01319 


\section{Contents}

1 Introduction 1

2 Jet algorithms and shapes at hadron colliders 5

$\begin{array}{lll}3 & \text { Factorized dijet cross section } & 7\end{array}$

4 Fixed-order $\mathcal{O}\left(\alpha_{s}\right)$ calculation of factorized components $\quad 8$

4.1 Jet functions 8

$\begin{array}{lll}4.2 & \text { Unmeasured beam functions } & 10\end{array}$

$\begin{array}{lll}4.3 \text { Soft function } & 10\end{array}$

$\begin{array}{ll}\text { 4.3.1 Calculation of the one-loop ingredients } & 11\end{array}$

$\begin{array}{lll}\text { 4.3.2 } & \text { Refactorization } & 14\end{array}$

5 RG evolution and the total NLL' cross section $\quad 15$

$\begin{array}{ll}5.1 \text { Hard function } & 15\end{array}$

$\begin{array}{ll}5.2 \text { Jet functions and unmeasured beam functions } & 16\end{array}$

$\begin{array}{lll}5.3 & \text { Soft function } & 17\end{array}$

$\begin{array}{lll}\text { 5.3.1 } & \text { Unmeasured evolution } & 17\end{array}$

$\begin{array}{lll}\text { 5.3.2 } & \text { Measured evolution } & 18\end{array}$

$\begin{array}{ll}\text { 5.3.3 Refactorized evolution } & 19\end{array}$

5.4 Total NLL' resummed cross section 21

5.5 A simple example 24

6 Conclusion 28

A Calculations of soft function components $\quad 31$

A.1 Beam-beam interference terms 31

A.2 Beam-jet interference terms $\quad 32$

A.3 Jet-jet interference terms 33

B Review of renormalization and RG evolution 34

\section{Introduction}

Jet production is associated with a large number of important scattering processes at colliders such as the Large Hadron Collider (LHC). It is therefore crucial to have a robust understanding of jets and jet production, and indeed much experimental and theoretical effort has gone into improving our understanding of jets. For hadron colliders, all theoretical predictions are based on the idea of QCD factorization [1, 2], which in its most 
basic form states that hadronic cross sections can be factorized into parton distribution functions (PDFs) and perturbatively calculable partonic cross sections. In multi-scale problems, these partonic cross sections can often be further factorized into pieces which only depend on a single scale and the renormalization group evolution (RGE) of each piece from the single scale that it is sensitive to (its "canonical scale") to a common scale resums the logarithms of ratios of these scales which would otherwise spoil the perturbative convergence of the partonic cross section when the scales are widely separated. An effective field theory approach to systematically factorizing cross sections is Soft-Collinear Effective Theory (SCET) [3-6].

A paradigmatic application of SCET is the factorization and resummation of logarithms in event shapes measured in $e^{+} e^{-}$collisions [7-10]. Such event shapes, denoted by $e$, can often be defined so that they vanish in the limit of perfectly narrow jets (so for example $e=0$ for the tree-level process $e^{+} e^{-} \rightarrow q \bar{q}$, and $e \rightarrow 0$ for events with additional radiation in the soft and collinear limits), and a fixed-order calculation of the cross section to $\mathcal{O}\left(\alpha_{s}^{n}\right)$ would then contain logarithms of the form $(1 / e) \alpha_{s}^{n} \ln ^{m} e$ (for $m \leq 2 n-1$ ). SCET factorization postulates that the partonic cross section can be written in terms a hard function $H$ which encapsulates the short-distance physics, jet functions $J$ that encapsulate collinear radiation within each jet and a soft function $S$ that encapsulates soft cross-talk between the jets, provided that the soft-collinear overlap (i.e. the 'zero-bin') has been properly subtracted from the jet functions [11]. For two back-to-back jets the factorization formula takes the schematic form

$$
d \sigma^{e^{+} e^{-}} \sim H(Q) \times J_{n}\left(Q e^{\alpha}\right) \otimes J_{\bar{n}}\left(Q e^{\alpha}\right) \otimes S_{n \bar{n}}(Q e),
$$

where $\otimes$ denotes a convolution over $e, n$ and $\bar{n}$ are the light-cone directions of the jets, the arguments of the functions denote the functions' canonical scales, $Q \sim \mathrm{E}_{\mathrm{cm}}$ is a shortdistance (hard) scale, and $\alpha$ is a parameter that depends on the choice of $e$ with $0<\alpha<1$ such that the canonical scales satisfy $Q e \ll Q e^{\alpha} \ll Q$ for $e \ll 1$. In the case of shapes which characterize multijet events (such as those of [12]), factorization simply involves more jet functions $J_{n_{i}}$ for each jet with direction $n_{i}$ and a more complicated soft function $S_{n_{1} n_{2} \cdots}$

One of the aims in the study of jet shapes is to study the internal energy patterns within a jet, i.e., the jet's substructure. This substructure can be used for example to help distinguish quark and gluon jets, or jets of purely QCD origin from those associated with other Standard Model mechanisms or from entirely new physics. Much work has recently been done on the analytical understanding of jet substructure, both for Monte Carlo event generator validation and for use as stand alone predictions [13-24].

Jet measurements at hadron colliders typically involve identifying jets of size $\mathcal{R}$ with the use of a jet algorithm, imposing a veto on the out-of-jet transverse momentum $p_{T}^{\text {cut }}$ for all radiation ${ }^{1}$ with (pseudo-)rapidity $y$ in the range $|y|<y_{\text {cut }}$ measured with respect to the beam axis. Such measurements are sensitive to hard scales (such as the Mandelstam variables $s, t, u$ in the case of dijet production) in addition to scales induced by the

\footnotetext{
${ }^{1}$ As discussed below, to the order we work this is the same as putting a veto on the third hardest jet.
} 
parameters $\mathcal{R}, y_{\text {cut }}$, and $p_{T}^{\text {cut }}$. When the substructure of jets is probed in the context of a jet measurement, additional scales such as $Q e$ and $Q e^{\alpha}$ for jet shapes are induced. Thus, there are not only scales associated with the substructure itself but also those associated with the more global context with which the probed jet was produced, and the large set of scales involved can span a wide range of energies.

Many of the ratios of these scales can be resummed using well known techniques such as SCET in similar ways to those described above for $e^{+} e^{-}$. In addition to the ingredients used in $e^{+} e^{-}$collisions, factorization formulae for hadronic collisions involve beam functions $B$ which account for initial-state radiation $[25,26]$, and we schematically have

$$
d \sigma^{p p} \sim H \times B \otimes \bar{B} \otimes J_{n_{1}} \otimes \cdots \otimes J_{n_{N}} \otimes S_{B \bar{B} n_{1} n_{2} \cdots} .
$$

While RGE of the functions appearing in eq. (1.2) resums a large set of logarithms, others, such as logarithms of $\mathcal{R}$ [27-29] and non-global logarithms (NGLs) [30-33], can present more of a challenge. Importantly, resummation of the jet size $\mathcal{R}$ has recently been explored in the context of subjets in [34] and in jet rates in the context of $e^{+} e^{-}$collisions in [35, 36], and in addition there has been progress in understanding NGLs both at fixed-order [37-40] and more recently a few novel approaches to understanding their all-orders resummation have been proposed [35, 41, 42].

In this paper we consider the case where the kinematics are such that NGLs are not enhanced and instead focus on resummation of logarithms of ratios of the dynamical scales associated with substructure (such as $Q e / Q$ and $Q e^{\alpha} / Q$ ) with fixed $p_{T}^{\text {cut }}, y_{\text {cut }}, \mathcal{R}$, and jet $p_{T}^{J}$. To this end, we restrict ourselves to the kinematic region

$$
\begin{aligned}
e^{-y_{\text {cut }}} & \ll 1 \\
p_{T}^{J} & \sim \sqrt{\hat{s}} \sim \sqrt{\hat{t}} \sim \sqrt{\hat{u}} \\
p_{T}^{\text {cut }} \mathcal{R}^{2} / p_{T}^{J} & \sim e \ll \mathcal{R}^{2} \ll 1 .
\end{aligned}
$$

Our approximations are valid to the order we work within about a decade of the value(s) of these parameters for which the NGLs are minimized. In the example we present, we have $e \sim \mathcal{O}\left(10^{-3}\right)$ in the peak region of the distribution and $\mathcal{R}^{2} \sim \mathcal{O}\left(10^{-1}\right)$, which means the leading NGLs, which are of the form $\alpha_{s}^{n} \ln ^{n}\left(p_{T}^{\text {cut }} \mathcal{R}^{2} / p_{T}^{J} e\right.$ ) (and first appear for $n \geq 2$ ), are not enhanced for $p_{T}^{\text {cut }} / p_{T}^{J} \sim \mathcal{O}\left(10^{-2}\right)$.

One class of event shapes that has been studied extensively in the literature and is the focus of the present work is that of angularities $\tau_{a}$, parameterized by a continuous variable $a$ (with $a<2$ for IR safety). The choice $a=0$ corresponds to the classic event shape thrust and $a=1$ corresponds to jet broadening. Angularities were originally defined in $[43,44]$ and studied in the context of SCET in [10, 45, 46]. In ref. [12], "jet shapes" 2 were defined by restricting the angularities to the constituents of a jet as defined by a jet algorithm (as opposed to all particles in the event) and were resummed to next-to-leading logarithmic (NLL) accuracy. In this work we consider a modified definition of angularities that is designed to be boost invariant about the colliding hadrons' axis, i.e., the beam axis.

\footnotetext{
${ }^{2}$ This is distinct from the jet shape as defined in [47, 48] and studied more recently in ref. [23, 49].
} 
We also note that the definition of the angularities we consider (which differs from that defined for $e^{+} e^{-}$colliders by a rescaling in the small $\tau_{a}$ limit) is such that the choice $a=0$ is closely related to the jet mass,

$$
\tau_{0}=m_{J}^{2} /\left(p_{T}^{J}\right)^{2}+\mathcal{O}\left(\tau_{0}^{2}\right) .
$$

Jet mass resummation has been studied indirectly by looking at the 1-jettiness global event shape [50] for single jet events in ref. [15], by using pQCD methods that neglect color interference effects in ref. [14], and in the threshold limit in refs. [13, 51], but to our knowledge has not been studied with the cuts described above, with full NLL' color interference effects, ${ }^{3}$ and in a manner that is valid away from the threshold limit. In addition, our results for $a=0$ can be straightforwardly extended to NNLL using the known anomalous dimensions together with the recently deduced two-loop unmeasured jet function anomalous dimension [36], which controls the evolution of both unmeasured jet and beam functions. In addition, we apply the refactorization procedure described in ref. [36] which allows the resummation of logarithms of $\mathcal{R}$ in the region described by eq. (1.3).

While we choose to study angularities as the choice of substructure observable, our basic setup is much more general. Indeed, we obtain many of the results specific to our choice of angularities by using identities that relate the jet functions and the observabledependent part of our soft function to analogous calculations in $e^{+} e^{-}$collisions. The part of the soft function that requires an entirely new calculation simply imposes the experimental $p_{T}^{\text {cut }}$ cut on radiation outside of the jets and the beams. This universal part of the soft function, labeled $\mathbf{S}^{\text {unmeas }}$, encapulates all the interjet cross-talk, and hence contains all perturbative information associated with real emission about the directions $n_{i}$ and the color flow. For each jet which has the angularity probed, which here and below we refer to (using the terminology of ref. [12]) as a "measured jet", we add a jet function and a soft function contribution that are both angularity dependent but color- and direction-trivial. Thus, other substructure measurements can be straightforwardly incorporated by substituting for their appropriate contributions at this step. If no measurement is performed on a jet (that is, the jet is identified but otherwise unprobed), which we refer to as an "unmeasured jet", only an unmeasured jet function (which we also present to $\mathcal{O}\left(\alpha_{s}\right)$ ) and $\mathbf{S}^{\text {unmeas }}$ are required. For dijet production, which is the focus of the current work, all four Wilson lines (those of the beams and the two jets) are confined to a plane, and the calculation of $\mathbf{S}^{\text {unmeas }}$ to $\mathcal{O}\left(\alpha_{s}\right)$ is tractable. In addition, the effect of different experimentally used vetoes, such as putting a $p_{T}^{\text {cut }}$ only on the third hardest jet (as opposed to all out-of-jet radiation) will only result in a difference in $\mathbf{S}^{\text {unmeas }}$ at $\mathcal{O}\left(\alpha_{s}^{2}\right)$ so our calculations apply there as well.

We also point out that while for unmeasured jets, the jet size $\mathcal{R}$ must scale with the SCET power counting parameter $\lambda$ and hence the requirement $\mathcal{R} \ll 1$ is essential, for measured jets this is not strictly needed since $\tau_{a} \ll 1$ is sufficient to ensure SCET kinematics. However, as we will see, both the jet algorithms and measurements simplify significantly

\footnotetext{
${ }^{3}$ For an explanation of which terms are included in our cross section by working to this order, see for example ref. [52].
} 
in this limit up to power corrections of the form $\mathcal{R}^{2}$ and $\tau_{a} / \mathcal{R}^{2}$, respectively, although we emphasize that the exact results can be obtained numerically using subtractions such as those of ref. [53]. Finally, we note that because there is no measurement on any radiation with $|y|<y_{\text {cut }}$, our factorization formulae will include "unmeasured beam functions", which to our knowledge have not appeared in the literature.

This paper is organized as follows. In section 2, we define the classes of jet algorithms and angularity definitions suitable for hadron colliders and relate them to the corresponding $e^{+} e^{-}$algorithms and angularities in the small $\mathcal{R}$ limit. In section 3 we outline the $2 \rightarrow 2$ kinematic relations needed for dijet production and discuss how both the Born cross section and the fully factorized and resummed SCET cross section are related to the basic building blocks that we then calculate to fixed order in section 4, namely the hard, jet, soft, and beam functions. We then use these results in section 5 to arrive at the NLL' resummed cross section for a generic $2 \rightarrow 2$ scattering channel both for when the jets are identified but otherwise left unmeasured (i.e., we are inclusive in the substructure properties) and for when the angularity of either (or both) jets is measured. From our calculations, one can obtain results for the case where the angularities of both jets $\tau_{a}^{1}$ and $\tau_{a}^{2}$ are separately measured (and by integrating, the case where $\tau_{a}^{1}+\tau_{a}^{2}$ is measured) as well as the cases where only one or neither are measured. For illustrative purposes, in our plots we focus on the case where both $\tau_{a}^{1}$ and $\tau_{a}^{2}$ are measured and $\tau_{a}^{1}=\tau_{a}^{2}$. Furthermore, we present explicit results for the simple channel $q q^{\prime} \rightarrow q q^{\prime}$ with different values of $\mathcal{R}$ and $p_{T}^{\text {cut }}$ and for several choices of the angularity parameter $a$, and demonstrate the reduction in scale uncertainty resulting from the refactorization techniques of [36]. We conclude in section 6 .

\section{Jet algorithms and shapes at hadron colliders}

The main difference between jet cross section measurements at $e^{+} e^{-}$colliders and hadron colliders is that the latter prefer observables that are invariant under boosts along the beam direction. The $k_{T}$-type algorithms used at the LHC (described in more detail in, for example, ref. [54]) merge particles successively using a pairwise metric

$$
\rho_{i j}=\min \left\{\left(p_{T}^{i}\right)^{2 p},\left(p_{T}^{j}\right)^{2 p}\right\} \frac{\Delta \mathcal{R}_{i j}^{2}}{\mathcal{R}^{2}},
$$

where $p=+1,0$, and -1 for the $k_{T}, \mathrm{C} / \mathrm{A}$, and anti- $k_{T}$ algorithms, respectively, $p_{T}^{i}$ is the transverse momentum (with respect to the beam) of particle $i, \mathcal{R}$ is a parameter characterizing the jet size, and

$$
\Delta \mathcal{R}_{i j} \equiv \sqrt{\left(\Delta y_{i j}\right)^{2}+\left(\Delta \phi_{i j}\right)^{2}}
$$

where $\Delta y_{i j}$ and $\Delta \phi_{i j}$ are the pseudo-rapidity and azimuthal angle differences of the particles measured with respect to the beam axis. Since pseudo-rapidities simply shift under boosts and azimuthal angles are invariant, $\Delta \mathcal{R}_{i j}$ is invariant under boosts along the beam direction. This pairwise metric is compared to the single particle metric of each particle, defined as

$$
\rho_{i}=\left(p_{T}^{i}\right)^{2 p}
$$


Two particles are merged if their pairwise metric is the smallest for the $(i j)$ pair over all particle pairs and is less than both of the single particle metrics, i.e., $\rho_{i j}<\min \left\{\rho_{i}, \rho_{j}\right\}$. This latter constraint amounts to

$$
\Delta \mathcal{R}_{i j}<\mathcal{R}
$$

In the following, we will work under the assumption that all particles in the jet are close to a jet axis at polar angle $\theta_{J}$ with respect to the beam axis such that $\Delta \mathcal{R}_{i j}$ can be expanded as

$$
\begin{aligned}
\Delta \mathcal{R}_{i j} & =\frac{1}{\sin \theta_{J}} \sqrt{\left(\Delta \theta_{i j}\right)^{2}+\sin ^{2} \theta_{J}\left(\Delta \phi_{i j}\right)^{2}}+\mathcal{O}\left(\left(\Delta \theta_{i j}\right)^{2},\left(\Delta \phi_{i j}\right)^{2}\right) \\
& =\frac{\theta_{i j}}{\sin \theta_{J}}+\mathcal{O}\left(\theta_{i j}^{2}\right),
\end{aligned}
$$

where in the first equality $\Delta \theta_{i j}$ and $\Delta \phi_{i j}$ are the angle differences in a spherical coordinate system with $\hat{z}$ in the beam axis direction, and $\theta_{i j}$ in the second equality is simply the angle between particles $i$ and $j$. This implies we can impose an $e^{+} e^{-}$-type polar angle restriction that particles are within a jet of size $R$ and rescale the results by

$$
R \rightarrow \mathcal{R} \sin \theta_{J}=\frac{\mathcal{R}}{\cosh y_{J}},
$$

where $y_{J}$ is the jet pseudo-rapidity, up to $\mathcal{O}\left(\mathcal{R}^{2}\right)$ corrections. This allows us to recycle many of the results of ref. [12]. The difference between our results and those obtained from the exact expression eq. (2.2) can be obtained numerically, e.g., with the methods of ref. [53], although the details are beyond the scope of the present work.

It is helpful to re-write the angularity definition used in ref. [12] in the context of $e^{+} e^{-}$ collisions in terms of ingredients that are boost invariant, such as $p_{T}$ and the right-hand side of eq. (2.5). To do so, first recall the definition used in terms of the pseudo-rapidities $y_{i J}$ and transverse momenta $p_{\perp}^{i J}$ of particles with respect to the jet axis,

$$
\tau_{a}^{e^{+} e^{-}}=\frac{1}{2 E_{J}} \sum_{i \in \mathrm{jet}}\left|p_{\perp}^{i J}\right| e^{-(1-a)\left|y_{i J}\right|}
$$

In the small angle approximation, we can write this as

$$
\tau_{a}^{e^{+} e^{-}}=\left(2 E_{J}\right)^{-(2-a)}\left(p_{T}\right)^{1-a} \sum_{i \in \text { jet }}\left|p_{T}^{i}\right|\left(\frac{\theta_{i J}}{\sin \theta_{J}}\right)^{2-a}\left(1+\mathcal{O}\left(\theta_{i J}^{2}\right)\right) .
$$

From the discussion above, all terms in the sum over particles are boost invariant. The one term that is not boost invariant is just the overall factor of $\left(2 E_{J}\right)^{2-a}$. Therefore, we can arrive at a boost invariant version of $\tau_{a}$ suitable for hadron colliders with a simple rescaling by a dimensionless factor,

$$
\begin{aligned}
\tau_{a} \equiv \tau_{a}^{p p} & \equiv \frac{1}{p_{T}} \sum_{i \in \text { jet }}\left|p_{T}^{i}\right|\left(\Delta \mathcal{R}_{i J}\right)^{2-a} \\
& =\left(\frac{2 E_{J}}{p_{T}}\right)^{2-a} \tau_{a}^{e^{+} e^{-}}+\mathcal{O}\left(\tau_{a}^{2}\right) .
\end{aligned}
$$


We emphasize again that the quantities on the right-hand side of the first line of eq. (2.9) are manifestly invariant under boosts along the beam axis, and that the second line allows us to recycle many of the results of ref. [12].

The one main difference between measurements done at $e^{+} e^{-}$colliders and hadron colliders that requires a novel calculation is the out-of-jet energy veto. In $e^{+} e^{-}$colliders, this is typically a cut on energy, whereas in hadron colliders it is typically a veto on transverse momentum: $p_{T}=E \sin \theta<p_{T}^{\text {cut }}$. This will require an entirely new soft function, which we present below.

\section{Factorized dijet cross section}

For dijet production at tree-level, momentum conservation implies that there are just three non-trivial variables to describe the final state at tree level, which we can take to be the jet (pseudo-) rapidities $y^{1,2}$ and the jet $p_{T}=\left|\mathbf{p}_{T}^{1}\right|=\left|\mathbf{p}_{T}^{2}\right|$. The momentum fractions of the incoming partons are related to these variables via

$$
x_{1,2}=\frac{2 p_{T}}{\mathrm{E}_{\mathrm{cm}}} \cosh \frac{\Delta y}{2} e^{ \pm Y},
$$

where $\Delta y=y_{1}-y_{2}$ is the rapidity difference of the two jets and $Y=\left(y_{1}+y_{2}\right) / 2$. The (partonic) Mandelstam variables can be written as

$$
\begin{aligned}
& s=4 p_{T}^{2} \cosh ^{2} \frac{\Delta y}{2} \\
& t=-2 p_{T}^{2} e^{\Delta y / 2} \cosh \frac{\Delta y}{2} \\
& u=-2 p_{T}^{2} e^{-\Delta y / 2} \cosh \frac{\Delta y}{2}=-s-t .
\end{aligned}
$$

The tree-level matrix element squared can be written as

$$
\left|\mathcal{M}_{\text {tree }}\right|^{2}=\operatorname{Tr}\left\{\mathbf{H}_{0} \mathbf{S}_{0}\right\}
$$

where $\mathbf{H}_{0}$ and $\mathbf{S}_{0}$ are the tree-level hard and soft functions, respectively, so the Born cross section takes form

$$
\frac{d \sigma_{\text {born }}}{d y_{1} d y_{2} d p_{T}}=\frac{p_{T}}{8 \pi x_{1} x_{2} E_{\mathrm{cm}}^{4}} \frac{1}{N} f_{1}\left(x_{1}, \mu\right) f_{2}\left(x_{2}, \mu\right) \operatorname{Tr}\left\{\mathbf{H}_{0} \mathbf{S}_{0}\right\}
$$

where $N$ is the normalization associated with averaging over initial particle quantum numbers (e.g., $N=4 N_{c}^{2}$ for quark scattering) and $f_{i}\left(x_{i}, \mu\right)$ is a PDF for parton $i$ with momentum fraction $x_{i}$.

The effect of radiative corrections to eq. (3.4) is described in the soft and collinear limits by higher-order hard, soft, beam, and jet functions. We consider the cases when both jets are unmeasured and when both jets are measured. When both jets are unmeasured the 
all-orders cross section takes the form

$$
\begin{aligned}
d \sigma \equiv & \frac{d \sigma}{d y_{1} d y_{2} d p_{T}} \\
= & \frac{p_{T}}{8 \pi x_{1} x_{2} E_{\mathrm{cm}}^{4}} \frac{1}{N} B\left(x_{1}, \mu\right) \bar{B}\left(x_{2}, \mu\right) \operatorname{Tr}\left\{\mathbf{H}(\mu) \mathbf{S}^{\text {unmeas }}(\mu)\right\} J_{1}(\mu) J_{2}(\mu) \\
& +\mathcal{O}\left(\alpha_{s} \mathcal{R}^{2}, \alpha_{s} e^{-2 y_{\text {cut }}}\right),
\end{aligned}
$$

where the $J_{i}(\mu)$ are unmeasured jet functions and $S^{\text {unmeas }}$ is the unmeasured soft function. When both jets are measured, the cross section takes the form

$$
\begin{aligned}
d \sigma\left(\tau_{a}^{1}, \tau_{a}^{2}\right) \equiv & \frac{d \sigma}{d y_{1} d y_{2} d p_{T} d \tau_{a}^{1} d \tau_{a}^{2}} \\
= & \frac{p_{T}}{8 \pi x_{1} x_{2} E_{\mathrm{cm}}^{4}} \frac{1}{N} B\left(x_{1}, \mu\right) \bar{B}\left(x_{2}, \mu\right) \operatorname{Tr}\left\{\mathbf{H}(\mu) \mathbf{S}\left(\tau_{a}^{1}, \tau_{a}^{2}, \mu\right)\right\} \otimes\left[J_{1}\left(\tau_{a}^{1}, \mu\right) J_{2}\left(\tau_{a}^{2}, \mu\right)\right] \\
& +\mathcal{O}\left(\alpha_{s} \tau_{a}^{i} / \mathcal{R}^{2}, \alpha_{s} e^{-2 y_{\mathrm{cut}}}\right)
\end{aligned}
$$

where $\otimes$ represents the two convolutions over the $\tau_{a}^{1,2}$. The case of a single measured jet, with the other jet unmeasured, is the obvious generalization of eqs. (3.5) and (3.6). The power corrections to eqs. (3.5) and (3.6) can be included via matching to fixed order QCD. Resummation of $\operatorname{logs}$ of $\tau_{a}$ is achieved by RG evolution of each factorized component from its canonical scale (cf. table 2) to the common scale $\mu$. Both the hard and soft function are in general matrices (which here and below we will refer to with bold face) which are hermitian and of rank $R$ equal to the number of linearly independent color operators associated with the hard process (e.g., $R=2$ for $q q \rightarrow q q, 3$ for $q q \rightarrow g g$, and 8 for $g g \rightarrow g g$ ). These operators mix under RG evolution which is accounted for with matrix RG equations. The fixed order calculation of the components in eqs. (3.5) and (3.6) and their RG evolution is the subject of the next sections.

\section{Fixed-order $\mathcal{O}\left(\alpha_{s}\right)$ calculation of factorized components}

\subsection{Jet functions}

In ref. [12], there are both "measured" and "unmeasured" jet functions, corresponding to jets whose angularity was measured as opposed to those that were identified but otherwise unprobed. The latter can be obtained using the hadron collider algorithms with the rescaling in eq. (2.6). We obtain

$$
J_{i}=1+\frac{\alpha_{s}}{2 \pi}\left[\left(\frac{C_{i}}{\epsilon^{2}}+\frac{\gamma_{i}}{\epsilon}\right)\left(\frac{\mu}{p_{T} \mathcal{R}}\right)^{2 \epsilon}+d_{J}^{i, \text { alg }}\right]
$$

where $i=q, g$ for quark and gluon jets (and $C_{i}$ is the Casimir invariant, $C_{q}=C_{F}$ and $C_{g}=C_{A}$ ), respectively, and

$$
\gamma_{q}=\frac{3 C_{F}}{2} \quad \gamma_{g}=\frac{\beta_{0}}{2}
$$


(with $\beta_{0}$ given in eq. (B.19)) and the finite corrections $d_{J}^{i, \text { alg }}$ are given in eqs. (A.19) and (A.30) of [12],

$$
\begin{gathered}
d_{J}^{i, \text { cone }}=2 \gamma_{i} \ln 2-C_{i} \frac{5 \pi^{2}}{12}+ \begin{cases}C_{F} \frac{7}{2} & \text { if } i=q \\
C_{A} \frac{137}{36}-T_{R} N_{f} \frac{23}{18} & \text { if } i=g\end{cases} \\
d_{J}^{i, k_{T}}=-C_{i} \frac{3 \pi^{2}}{4}+ \begin{cases}C_{F} \frac{13}{2} & \text { if } i=q \\
C_{A} \frac{67}{9}-T_{R} N_{f} \frac{23}{9} & \text { if } i=g\end{cases}
\end{gathered}
$$

where $d_{J}^{i, k_{T}}$ is the same constant for all $k_{T}$-type algorithms $\left(k_{T}\right.$, anti- $k_{T}$, and $\left.\mathrm{C} / \mathrm{A}\right)$.

For measured jet functions, we need to apply the rescaling eq. (2.9). The identity

$$
A^{-1} \delta\left(A^{-1} \tau-\hat{\tau}\right)=\delta(\tau-A \hat{\tau})
$$

implies that this rescaling can be accomplished to all orders via the transformation

$$
J_{i}\left(\tau_{a}\right)=\left(\frac{p_{T}}{2 E_{J}}\right)^{2-a} J_{i}^{e^{+}} e^{-}\left(\left(\frac{p_{T}}{2 E_{J}}\right)^{2-a} \tau_{a}\right)
$$

where $J_{i}^{e^{+} e^{-}}\left(\tau_{a}\right)$ is the jet function of [12]. This gives

$$
J_{i}\left(\tau_{a}\right)=\left.J_{i}^{e^{+} e^{-}}\left(\tau_{a}\right)\right|_{2 E_{J} \rightarrow p_{T}}
$$

i.e., it is simply obtained from $J_{i}^{e^{+} e^{-}}\left(\tau_{a}\right)$ by making the replacement $2 E_{J} \rightarrow p_{T}$. These can be obtained for the quark case from ref. [46] and for the gluon case by performing the integral in eq. (4.22) of ref. [12] after setting $\Theta_{\text {alg }}(x) \rightarrow 0$ which is valid to $\mathcal{O}\left(\tau_{a} / \mathcal{R}^{2}\right)$. We record the results here as

$$
J_{i}\left(\tau_{a}\right)=\delta\left(\tau_{a}\right)-\frac{\alpha_{s}}{2 \pi}\left[\left(\frac{\mu}{p_{T}}\right)^{2 \epsilon}\left(\frac{1}{\tau_{a}}\right)^{1+\frac{2 \epsilon}{2-a}}\left(\frac{1}{\epsilon} \frac{2 C_{i}}{1-a}+\frac{\gamma_{i}}{1-a / 2}\right)-\delta\left(\tau_{a}\right) f_{i}(a)\right],
$$

where

$$
\begin{aligned}
f_{q}(a)=\frac{2 C_{F}}{1-a / 2}[ & \frac{7-13 a / 2}{4}-\frac{\pi^{2}}{12} \frac{3-5 a+9 a^{2} / 4}{1-a} \\
& \left.-\int_{0}^{1} d x \frac{1-x+x^{2} / 2}{x} \ln \left[x^{1-a}+(1-x)^{1-a}\right]\right] \\
f_{g}(a)=\frac{1}{1-a / 2}[ & C_{A}\left((1-a)\left(\frac{67}{18}-\frac{\pi^{2}}{3}\right)+\frac{\pi^{2}}{6} \frac{(1-a / 2)^{2}}{1-a}\right. \\
& \left.-\int_{0}^{1} d x \frac{(1-x(1-x))^{2}}{x(1-x)} \ln \left[x^{1-a}+(1-x)^{1-a}\right]\right) \\
& \left.-T_{R} N_{f}\left(\frac{20-23 a}{18}-\int_{0}^{1} d x(2 x(1-x)-1) \ln \left[x^{1-a}+(1-x)^{1-a}\right]\right)\right] .
\end{aligned}
$$

Finally, we note that the integral over $\tau_{a}$ of the measured jet function is not simply related to the unmeasured jet function and refer the reader to ref. [36] for a detailed explanation. 


\subsection{Unmeasured beam functions}

While the unmeasured beam function has not to our knowledge appeared in the literature, it is directly related to the unmeasured fragmenting jet function of [55]. The unmeasured fragmenting jet function for a jet of energy $E$ and $\left(e^{+} e^{-}\right)$cone radius $R$ can be written as

$$
\mathcal{G}(E, R, z, \mu)=\sum_{i} \int \frac{d z^{\prime}}{z^{\prime}} \mathcal{J}_{i j}\left(E, R, z^{\prime}, \mu\right) D_{j}^{h}\left(z / z^{\prime}, \mu\right)+\mathcal{O}\left(\Lambda_{\mathrm{QCD}}^{2} / E^{2}\right),
$$

where $D_{i}^{h}(x, \mu)$ is a fragmentation function for parton $i$ in hadron $h$ and the $\mathcal{J}_{i j}$ are matching coefficients which are given in eq. (5) of ref. [55]. The dependence on $E$ and $R$ in $\mathcal{J}_{i j}$ (at least to $\mathcal{O}\left(\alpha_{s}\right)$ ) is such that we can write

$$
\mathcal{J}_{i j}\left(E, R, z^{\prime}, \mu\right) \equiv \mathcal{J}_{i j}\left(2 E \tan \frac{R}{2}, z^{\prime}, \mu\right)
$$

i.e., $E$ and $R$ always appear in the combination $E \tan \frac{R}{2}$. Using the crossing relations of section IIIC of ref. [56], it can be shown that an unmeasured beam function in a collider with center-of-mass energy $\mathrm{E}_{\mathrm{cm}}$ and a rapidity cut of $y_{\text {cut }}$ can be written as

$$
\begin{aligned}
B_{i}\left(x_{i}, \mu\right) & \equiv B_{i}\left(\mathrm{E}_{\mathrm{cm}}, y_{\mathrm{cut}}, x_{i}, \mu\right) \\
& =\sum_{j} \int \frac{d z}{z} \mathcal{J}_{i j}\left(x_{i} \mathrm{E}_{\mathrm{cm}} e^{-y_{\mathrm{cut}}}, z, \mu\right) f_{j}\left(x_{i} / z, \mu\right)+\mathcal{O}\left(\Lambda_{\mathrm{QCD}}^{2} / E^{2}\right)
\end{aligned}
$$

where $\mathcal{J}_{i j}$ are the same matching coefficients as in eq. (4.10), at least to $\mathcal{O}\left(\alpha_{s}\right),{ }^{4}$ and we used the correspondence between an $e^{+} e^{-}$jet and a beam with label momentum $x_{i} \mathrm{E}_{\mathrm{cm}}$ and rapidity cut $y_{\text {cut }}$

$$
E \tan \frac{R}{2} \rightarrow x_{i} \mathrm{E}_{\mathrm{cm}} e^{-y_{\mathrm{cut}}},
$$

which is valid up to $\mathcal{O}\left(e^{-2 y_{\text {cut }}}\right)$ corrections. For the dijet cross section we consider, the $x_{i}$ are fixed via eq. (3.1).

\subsection{Soft function}

In general, we can write the bare soft function at $\mathcal{O}\left(\alpha_{s}\right)$ for dijet production when both jets have $\tau_{a}$ measured as

$$
\mathbf{S}\left(\tau_{a}^{1}, \tau_{a}^{2}\right)=\mathbf{S}^{\text {unmeas }} \delta\left(\tau_{a}^{1}\right) \delta\left(\tau_{a}^{2}\right)+\left[\mathbf{S}_{0} S^{\text {meas }}\left(\tau_{a}^{1}\right) \delta\left(\tau_{a}^{2}\right)+(1 \leftrightarrow 2)\right]+\mathcal{O}\left(\alpha_{s}^{2}\right),
$$

where $\mathbf{S}^{\text {unmeas }}=\mathbf{S}_{0}+\mathcal{O}\left(\alpha_{s}\right)$ is the part of the soft function that is always present (both when the jets are measured and unmeasured). The bare soft function is $\mu$ independent, and we will distinguish the corresponding renormalized function with an explicit argument $\mu$. In the cases that neither of the jets or only one jet is measured, the corresponding $S^{\text {meas }}$ pieces on the right-hand are simply not included, while $\mathbf{S}^{\text {unmeas }}$ is always included. For more jets, the result can be extended straightforwardly, although our explicit results only apply to planar jet configurations (as is necessarily the case for dijet production).

\footnotetext{
${ }^{4}$ It is argued in [57] that measured beam and jet functions have the same anomalous dimension to all orders (at least for the measured case), but since the PDFs and fragmentation functions differ perturbatively at $\mathcal{O}\left(\alpha_{s}^{2}\right)$ [58] the matching coefficients must differ for the beam and jet functions starting at this order.
} 


\subsubsection{Calculation of the one-loop ingredients}

The part of the soft function corresponding to the measurement of $\tau_{a}^{i}$ on jet $i, S^{\text {meas }}\left(\tau_{a}^{i}\right)$, is obtained from summing over the interference of jet $i$ with all other jets and the beams. Contributions from radiation arising from the interference of jets/beams $j$ and $k$ with $j, k \neq i$ give power corrections in $\mathcal{R}$. The calculation of $S^{\text {meas }}\left(\tau_{a}^{i}\right)$ can be obtained from the results for $S_{i j}^{\text {meas }}\left(\tau_{a}^{i}\right)$ given in eq. (5.18) of ref. [12] through the rescaling in eq. (2.9). We find

$$
\begin{aligned}
S^{\text {meas }}\left(\tau_{a}^{i}\right) & =2 \sum_{i<j}\left(\frac{p_{T}}{2 E_{J}}\right)^{2-a} S_{i j}^{\text {meas }}\left(\left(\frac{p_{T}}{2 E_{J}}\right)^{2-a} \tau_{a}^{i}\right) \\
& =\frac{1}{\epsilon} \frac{\alpha_{s} C_{i}}{\pi} \frac{e^{\gamma_{E} \epsilon}}{\Gamma(1-\epsilon)} \frac{1}{1-a}\left(\frac{1}{\tau_{a}^{i}}\right)^{1+2 \epsilon}\left(\frac{\mu}{p_{T}}\right)^{2 \epsilon} \mathcal{R}^{2 \epsilon(1-a)},
\end{aligned}
$$

which clearly has the desired boost-invariant properties.

The additional part of the soft function we require, $\mathbf{S}^{\text {unmeas }}$, can be written as a sum of contributions in the same manner as ref. [12],

$$
\mathbf{S}^{\text {unmeas }}=\mathbf{S}_{0}+\left[\mathbf{S}_{0} \sum_{i<j} \mathbf{T}_{i} \cdot \mathbf{T}_{j}\left(S_{i j}^{\mathrm{incl}}+\sum_{k=1}^{N} S_{i j}^{k}\right)+\text { h.c. }\right] \text {, }
$$

where h.c. denotes the hermitian conjugate. Here, we use the color space formalism as described in refs. $[59,60]$. The $4 ! /(2 !)^{2}=6$ matrices $\mathbf{T}_{i} \cdot \mathbf{T}_{j}$ are of rank $R$, the same as that of $\mathbf{S}_{0}$, and account for the mixing of color operators in a given basis into each other at $\mathcal{O}\left(\alpha_{s}\right)$. The difference from ref. [12] is that now each contribution involves a $p_{T}$ veto instead of an energy veto as well as a different jet algorithm. In particular, defining

$$
\begin{aligned}
\Theta_{p_{T}} & \equiv \Theta\left(k^{0} \sin \theta_{k B}<p_{T}^{\text {cut }}\right) \\
\Theta_{\mathcal{R}}^{k} & \equiv \Theta\left(\mathcal{R}_{k J}<\mathcal{R}\right),
\end{aligned}
$$

we now have

$$
S_{i j}^{\text {incl }} \equiv \frac{1}{\epsilon} \frac{\alpha_{s}}{2 \pi}\left(\frac{\mu}{p_{T}^{\text {cut }}}\right)^{2 \epsilon} \mathcal{I}_{i j}^{\text {incl }}=-g^{2} \mu^{2 \epsilon} \int \frac{d^{d} k}{(2 \pi)^{d-1}} \frac{n_{i} \cdot n_{j}}{\left(n_{i} \cdot k\right)\left(n_{j} \cdot k\right)} \delta\left(k^{2}\right) \Theta\left(k^{0}\right) \Theta_{p_{T}},
$$

and

$$
S_{i j}^{k} \equiv \frac{1}{\epsilon} \frac{\alpha_{s}}{2 \pi}\left(\frac{\mu}{p_{T}^{\text {cut }}}\right)^{2 \epsilon} \mathcal{I}_{i j}^{k}=g^{2} \mu^{2 \epsilon} \int \frac{d^{d} k}{(2 \pi)^{d-1}} \frac{n_{i} \cdot n_{j}}{\left(n_{i} \cdot k\right)\left(n_{j} \cdot k\right)} \delta\left(k^{2}\right) \Theta\left(k^{0}\right) \Theta_{p_{T}} \Theta_{\mathcal{R}}^{k},
$$

where $i, j$, and $k$ can each be either of the beams or one of the jets (with $i \neq j$ ).

We first perform the energy and trivial parts of the angular integration of eq. (4.18) for generic $i, j$ (either jet or beam). To do this, we align the 1-direction (or " $\hat{z} ")$ with direction $\vec{n}_{i}$ and put the $\vec{n}_{j}$ vector in the 12-plane, and the beam direction $\vec{n}_{B}$ in the 123-spatial part of $d$-dimensional space. Using the shorthands $c_{i j} \equiv 1-n_{i} \cdot n_{j}, s_{i j} \equiv\left(1-c_{i j}^{2}\right)^{1 / 2}, c_{i} \equiv \cos \theta_{i}$, 
and $s_{i} \equiv \sin \theta_{i}$, the dot products of the gluon's 3 -momentum, $\vec{k}$, with these unit vectors take the form

$$
\begin{aligned}
\vec{n}_{i} \cdot \vec{k} & =c_{1} \\
\vec{n}_{j} \cdot \vec{k} & =c_{i j} c_{1}+s_{i j} s_{1} c_{2} \\
\vec{n}_{B} \cdot \vec{k} & =n_{B 1} c_{1}+n_{B 2} s_{1} c_{2}+n_{B 3} s_{1} s_{2} c_{3}
\end{aligned}
$$

for the $i, j$, and beam directions, respectively. In this frame, $\mathcal{I}_{i j}^{\text {incl }}$ takes the form (in $\overline{\mathrm{MS}}$ )

$$
\begin{aligned}
\mathcal{I}_{i j}^{\text {incl }}= & \frac{\left(1-c_{i j}\right) e^{\gamma_{E} \epsilon}}{2 \sqrt{\pi} \Gamma(1 / 2-\epsilon)} \int_{0}^{\pi} d \theta_{1} \sin ^{1-2 \epsilon} \theta_{1} \int_{0}^{\pi} d \theta_{2} \sin ^{-2 \epsilon} \theta_{2} \frac{1}{1-c_{1}} \frac{1}{1-c_{i j} c_{1}-s_{i j} s_{1} c_{2}} \\
& \times\left[\frac{\Gamma(1 / 2-\epsilon)}{\sqrt{\pi} \Gamma(-\epsilon)} \int_{0}^{\pi} d \theta_{3} \sin ^{-1-2 \epsilon} \theta_{3}\left(1-\left(n_{B 1} c_{1}+n_{B 3} s_{1} c_{2}+n_{B 3} s_{1} s_{2} c_{3}\right)^{2}\right)^{\epsilon}\right] .
\end{aligned}
$$

The quantity in parenthesis to the $\epsilon^{1}$ power in the second line is the square of the sine of the gluon-beam angle and comes from doing the $k^{0}$ (energy) integral over the $p_{T}$ veto, $\Theta_{p_{T}}$. For planar events (such as dijet events at hadron colliders), $n_{B 3}=0$ (since the beam is in the $i j$-plane for all $i, j$ ) and the integration over $\theta_{3}$ can be easily performed. The entire second line (the quantity in brackets) then becomes simply

$$
[\cdots] \stackrel{\text { planar }}{\longrightarrow}\left(1-\left(n_{B 1} c_{1}+n_{B 2} s_{1} c_{2}\right)^{2}\right)^{\epsilon},
$$

with $n_{B 2}^{2}=1-n_{B 1}^{2}$. We also note that when $i$ is equal to the beam direction (so $n_{B 1}=1$ and $n_{B 2}=0$ ), this quantity reduces to

$$
[\cdots] \stackrel{n_{i}=n_{B}}{\longrightarrow} \sin ^{2 \epsilon} \theta_{1}
$$

In this case, the $\epsilon$ dependence in the overall power of $\sin \theta_{1}$ cancels and we are left with a divergence unregulated by dimensional regularization. This is the well-known rapidity divergence that is present for a $p_{T}$ veto. This can be treated within the context of $\mathrm{SCET}_{\mathrm{II}}$ as was done for example in ref. [61]. Here, we will opt instead to veto on radiation only below a rapidity cut $y_{\text {cut }}$ which is consistent with what is done at the LHC since radiation going down the beam pipes is not measured. We compute the soft function components $\mathcal{I}_{i j}^{i}$ and $\mathcal{I}_{i j}^{\text {incl }}$ for the case $i$ and $j$ can each either be beams or jets in appendix A and record the results in table 1. For the case that either $i$ or $j$ is a beam, we only compute the full out-of-beam contribution, e.g. $\mathcal{I}_{J B}^{\text {incl }}+\mathcal{I}_{J B}^{B}\left(\right.$ or $\mathcal{I}_{B \bar{B}}^{\text {incl }}+\mathcal{I}_{B \bar{B}}^{B}+\mathcal{I}_{B \bar{B}}^{\bar{B}}$ for the case both $i$ and $j$ are beams) to avoid having to regulate the rapidity divergences in individual components.

For several of the components, we use the fact that the result is boost invariant along the beam direction to boost to the frame where the jets are back-to-back. The relation between the back-to-back frame beam-jet angle $\theta_{J}$ and the jet rapidities in the lab frame is

$$
\cos \theta_{J}=\tanh \frac{\Delta y}{2}
$$




\begin{tabular}{|c|c|}
\hline Contribution & Result \\
\hline $\mathcal{I}_{B \bar{B}}^{\text {incl }}+\mathcal{I}_{B \bar{B}}^{B}+\mathcal{I}_{B \bar{B}}^{\bar{B}}$ & $2 y_{\text {cut }}$ \\
$\mathcal{I}_{B \bar{B}}^{1}+\mathcal{I}_{B \bar{B}}^{2}$ & $\mathcal{O}\left(\mathcal{R}^{2}\right)$ \\
\hline $\mathcal{I}_{B J}^{\text {incl }}+\mathcal{I}_{B J}^{B}+\mathcal{I}_{B J}^{\bar{B}}$ & $-\frac{1}{2 \epsilon}+y_{\text {cut }}-y_{J}+\epsilon \frac{\pi^{2}}{24}$ \\
$\mathcal{I}_{B J}^{J}$ & $\frac{1}{2 \epsilon} \mathcal{R}^{-2 \epsilon}\left(1-\epsilon^{2} \frac{\pi^{2}}{12}\right)$ \\
$\mathcal{I}_{B J}^{k \neq J, B}$ & $\mathcal{O}\left(e^{-y_{\text {cut }}}, \mathcal{R}^{2}\right)$ \\
\hline $\mathcal{I}_{12}^{\text {incl }}$ & $\left(2 \cosh \frac{\Delta y}{2}\right)^{-2 \epsilon}\left(-\frac{1}{\epsilon}+\frac{\epsilon}{2}(\Delta y)^{2}+\epsilon \frac{\pi^{2}}{12}\right)$ \\
$\mathcal{I}_{12}^{1}+\mathcal{I}_{12}^{2}$ & $\frac{1}{\epsilon} \mathcal{R}^{-2 \epsilon}\left(1-\epsilon^{2} \frac{\pi^{2}}{12}\right)$ \\
$\mathcal{I}_{12}^{B, \bar{B}}$ & $\mathcal{O}\left(e^{-y_{\text {cut }}}\right)$ \\
\hline
\end{tabular}

Table 1. A summary of results for the "unmeasured" part of the soft function, $S^{\text {unmeas }}$, up to $\mathcal{O}\left(e^{-y_{\text {cut }}}, \mathcal{R}^{2}\right)$. Here, the subscript $J$ refers to the two jets, $J=1,2$, and $B$ and $\bar{B}$ refer to the two beams, and $\Delta y=y_{1}-y_{2}$. Each component is explicitly boost invariant about the beam direction (with $2 y_{\text {cut }}$ in the $B-\bar{B}$ interference terms in general given by the rapidity difference of the forward and backward beam cuts).

where $\Delta y=y_{1}-y_{2}$ is the rapidity difference of the two jets. This also means that when putting a polar angle restriction on the emitted gluon in the back-to-back frame, one has to apply the correspondence eq. (4.24) in using eq. (2.6), which amounts to the replacement

$$
\tan \frac{R}{2} \rightarrow \frac{\mathcal{R}}{2 \cosh \Delta y / 2}
$$

where dependence on the left-hand side arises from enforcing a restriction on the polar angle of the gluon about a jet $(\theta<R)$ in the back-to-back frame.

Using the color algebra identity $\sum_{i} \mathbf{T}_{i}=0$ and the kinematic relations

$$
\begin{aligned}
& \ln \frac{n_{J} \cdot n_{B}}{2}=-y_{J}-\ln \left(2 \cosh y_{J}\right) \\
& \ln \frac{n_{J} \cdot \bar{n}_{B}}{2}=y_{J}-\ln \left(2 \cosh y_{J}\right),
\end{aligned}
$$

for jets $J=1,2$, and

$$
\ln \frac{n_{1} \cdot n_{2}}{2}=\ln \frac{(2 \cosh \Delta y / 2)^{2}}{\left(2 \cosh y_{1}\right)\left(2 \cosh y_{2}\right)}
$$

we find

$$
\begin{aligned}
\mathbf{S}^{\text {unmeas }}=\mathbf{S}_{0}+\frac{\alpha_{s}}{\pi}\left\{\mathbf{S}_{0}[\right. & \left(\frac{1}{2 \epsilon}+\ln \frac{\mu}{p_{T}^{\text {cut }}}\right)\left(\mathbf{S}^{\mathrm{div}}+\sum_{i=1,2} C_{i} \ln \mathcal{R}\right)-\frac{1}{2} \sum_{i=1,2} C_{i} \ln ^{2} \mathcal{R} \\
& \left.\left.-\mathbf{T}_{1} \cdot \mathbf{T}_{2} \ln \left(1+e^{\Delta y}\right) \ln \left(1+e^{-\Delta y}\right)\right]+ \text { h.c. }\right\}+\mathcal{O}\left(\alpha_{s}^{2}\right) .
\end{aligned}
$$

In this equation,

$$
\begin{aligned}
\mathbf{S}^{\mathrm{div}} & =\sum_{i<j} \mathbf{T}_{i} \cdot \mathbf{T}_{j} \ln \frac{n_{i} \cdot n_{j}}{2}-y_{\text {cut }}\left(C_{B}+C_{\bar{B}}\right)-\sum_{i=1,2} C_{i} \ln \left(2 \cosh y_{i}\right) \\
& =\Delta \gamma_{s s}\left(m_{i}\right)-\mathbf{M}^{\prime}\left(m_{i}\right)
\end{aligned}
$$


where in the second line we wrote the result in terms two functions defined by

$$
\begin{aligned}
\Delta \gamma_{s s}\left(m_{i}\right) & =\sum_{i=B, \bar{B}} C_{i} \ln \frac{x_{i} \mathrm{E}_{\mathrm{cm}} e^{-y_{\mathrm{cut}}}}{m_{i}}+\sum_{i=1,2} C_{i} \ln \frac{p_{T}}{m_{i}} \\
\mathbf{M}^{\prime}\left(m_{i}\right) & \equiv-\sum_{i<j} \mathbf{T}_{i} \cdot \mathbf{T}_{j} \ln \frac{s_{i j}}{m_{i} m_{j}}
\end{aligned}
$$

where $s_{i j} \equiv 2 p_{i} \cdot p_{j}>0$ (and where $p_{i}=x_{i} \mathrm{E}_{\mathrm{cm}}$ for the beams $i=B, \bar{B}$ ). Note that for later convenience we have defined these functions so that each separately depends on a set of parameters $m_{i}$. The dependence on $m_{i}$ cancels in the sum in the second line of eq. (4.29).

\subsubsection{Refactorization}

We note here that one can also construct the ingredients needed for the refactorized cross section as was done in ref. [36] for the resummation of (global) logs of $\mathcal{R}$ from the ingredients in table 1. In particular, the conclusions of ref. [36] suggest that $\mathbf{S}^{\text {unmeas }}$ should be factorized as

$$
\begin{aligned}
\mathbf{S}^{\text {unmeas }} & =\frac{1}{2} \mathbf{S}_{0} \int_{0}^{p_{T}^{\text {cut }}} d E\left[\mathbf{S}_{s}(E) \otimes s_{s c}^{1}(E \mathcal{R}) \otimes s_{s c}^{2}(E \mathcal{R})\right]+\text { h.c. } \\
& =\mathbf{S}_{0}+\frac{\alpha_{s}}{4 \pi} \frac{1}{2}\left[\mathbf{S}_{0}\left(\mathbf{S}_{s}^{(1)}\left(p_{T}^{\text {cut }}\right)+\sum_{k=1,2} S_{s c}^{k(1)}\left(p_{T}^{\text {cut }} \mathcal{R}\right)\right)+\text { h.c. }\right]+\mathcal{O}\left(\alpha_{s}^{2}\right),
\end{aligned}
$$

where $\otimes$ is a convolution over the variable $E$ and the functions $\mathbf{S}_{s}$ and $S_{s c}^{k}$ are the global soft (with radiation anywhere except for the beams) and soft-collinear (with radiation within jet $k$ ) functions, respectively, and where

$$
\begin{aligned}
\mathbf{s}_{s}\left(p_{T}^{\text {cut }}\right) & \equiv \frac{d}{d p_{T}^{\text {cut }}} \mathbf{S}_{s}\left(p_{T}^{\text {cut }}\right) \\
s_{s c}^{k}\left(p_{T}^{\text {cut }} \mathcal{R}\right) & \equiv \frac{d}{d p_{T}^{\text {cut }}} S_{s c}^{k}\left(p_{T}^{\text {cut }} \mathcal{R}\right)
\end{aligned}
$$

with both functions $f=\mathbf{S}_{s}, S_{s c}^{k}$ normalized as $f(x)=\theta(x)+\sum_{i=1}\left(\frac{\alpha_{s}}{4 \pi}\right)^{n} f^{(n)}(x)$. Note that all of the non-trivial color mixing occurs in $\mathbf{S}_{s}$. This is due to the fact that the soft-collinear modes of refs. $[35,36]$ are confined to a single jet and is expected to hold to all orders.

In terms of the ingredients in table 1 , we have

$$
\begin{aligned}
\mathbf{S}_{s}^{(1)}\left(p_{T}^{\text {cut }}\right) & =\frac{4}{\epsilon}\left(\frac{\mu}{p_{T}^{\text {cut }}}\right)^{2 \epsilon} \sum_{i<j} \mathbf{T}_{i} \cdot \mathbf{T}_{j}\left[\mathcal{I}_{i j}^{\text {incl }}+\left(\delta_{i B}+\delta_{i \bar{B}}\right)\left(\delta_{j J_{1}}+\delta_{j J_{2}}\right) \mathcal{I}_{i j}^{i}+\delta_{i B} \delta_{i \bar{B}}\left(\mathcal{I}_{i j}^{i}+\mathcal{I}_{i j}^{j}\right)\right] \\
& =\frac{4}{\epsilon}\left(\frac{\mu}{p_{T}^{\text {cut }}}\right)^{2 \epsilon}\left[\sum_{i=1,2} \frac{C_{i}}{2 \epsilon}\left(1-\epsilon^{2} \frac{\pi^{2}}{12}\right)+\mathbf{S}^{\text {div }}-2 \epsilon \mathbf{T}_{1} \cdot \mathbf{T}_{2} \ln \left(1+e^{\Delta y}\right) \ln \left(1+e^{-\Delta y}\right)\right]
\end{aligned}
$$

and

$$
S_{s c}^{k(1)}\left(p_{T}^{\text {cut }} \mathcal{R}\right)=\frac{4}{\epsilon}\left(\frac{\mu}{p_{T}^{\text {cut }}}\right)^{2 \epsilon} \sum_{i<j} \mathbf{T}_{i} \cdot \mathbf{T}_{j}\left[\delta_{i k} \mathcal{I}_{i j}^{i}\right]=\frac{4}{\epsilon}\left(\frac{\mu}{p_{T}^{\text {cut }} \mathcal{R}}\right)^{2 \epsilon}\left[-\frac{C_{k}}{2 \epsilon}\left(1-\epsilon^{2} \frac{\pi^{2}}{12}\right)\right] .
$$




\section{RG evolution and the total NLL' cross section}

In this section, we apply Renormalization Group ( $R G$ ) methods to the functions calculated in this paper and arrive at the result for the total NLL' resummed cross section. These functions can be divided into those which are multiplicatively renormalized and those that renormalize via a convolution. The former include the hard function and unmeasured jet functions and the unmeasured part of the soft function, and the latter includes measured jet and soft functions.

\subsection{Hard function}

The hard function $\mathbf{H}$ for $N-2$ jet production in hadron collisions is a matrix in color space with rank $R$ (the same as that of the soft function). It can be written in terms of Wilson coefficients $C_{i}$ as $(\mathbf{H})_{i j}=C_{i} C_{j}^{*}$, each of which mix into each other under renormalization, i.e., $C_{i}^{\text {bare }}=\sum_{j}\left(\mathbf{Z}_{H}(\mu)\right)_{i j} C_{j}$ which implies that

$$
\mathbf{H}^{\text {bare }}=\mathbf{Z}_{H}(\mu) \mathbf{H}(\mu) \mathbf{Z}_{H}^{\dagger}(\mu) .
$$

The $\mu$-independence of the left-hand side of eq. (5.1) implies that $\mathbf{H} \equiv \mathbf{H}(\mu)$ obeys the RGE

$$
\frac{d \mathbf{H}}{d \ln \mu}=\boldsymbol{\Gamma}_{H} \mathbf{H}+\mathbf{H} \boldsymbol{\Gamma}_{H}^{\dagger},
$$

where

$$
\boldsymbol{\Gamma}_{H} \equiv-\mathbf{Z}_{H}^{-1} \frac{d}{d \ln \mu} \mathbf{Z}_{H}
$$

This RGE preserves the hermiticity of $\mathbf{H}$ under RG evolution. $\boldsymbol{\Gamma}_{H}$ in eq. (5.2) is given (to $\left.\mathcal{O}\left(\alpha_{s}^{2}\right)\right)$ by $[62,63]$

$$
\boldsymbol{\Gamma}_{H}=\frac{1}{2} \sum_{i=1}^{N}\left[C_{i} \Gamma_{c}\left(\alpha_{s}\right) \ln \frac{m_{i}^{2}}{\mu^{2}}-\frac{\alpha_{s}}{\pi} \gamma_{i}\right]+\Gamma_{c}\left(\alpha_{s}\right) \mathbf{M}\left(m_{i}\right),
$$

where $\gamma_{i}$ is given in eq. (4.2), $\Gamma_{c}\left(\alpha_{s}\right)$ is the cusp anomalous dimension (given in eq. (B.20)), and $m_{i}$ is an arbitrary parameter(s) which can be chosen for convenience and can be shown to cancel between the first term and $\mathbf{M}\left(m_{i}\right)$. The first term is (implicitly) proportional to an identity matrix and $\mathbf{M}$ in the second term involvers a non-trivial matrix of rank $R$, which can be written as

$$
\begin{aligned}
\mathbf{M}\left(m_{i}\right) & \equiv-\sum_{i<j} \mathbf{T}_{i} \cdot \mathbf{T}_{j}\left[\ln \left((-1)^{\Delta_{i j}} \frac{s_{i j}}{m_{i} m_{j}}-i 0^{+}\right)\right] \\
& =\mathbf{M}^{\prime}\left(m_{i}\right)+i \pi \mathbf{T}
\end{aligned}
$$

where $\Delta_{i j}$ is 0 for beam-jet interference and 1 for beam-beam and jet-jet interference, $s_{i j}=2 p_{i} \cdot p_{j}>0$, and in the second line we explicitly separated the terms of the form $\Delta_{i j} \ln (-1)$ into the matrix $i \pi \mathbf{T}$, where

$$
\mathbf{T} \equiv \sum_{i<j} \Delta_{i j} \mathbf{T}_{i} \cdot \mathbf{T}_{j}
$$


and $\mathbf{M}^{\prime}\left(m_{i}\right)$ is defined in eq. (4.30). The matrix $\mathbf{M}$ is worked out for a set of choices of color bases for all $2 \rightarrow 2$ channels in ref. [64] with the choice $m_{i}^{2}=-t>0$ (the Mandelstam variable) in the $q q^{\prime} \rightarrow q q^{\prime}$ channel (and the choice for other channels obtained by crossing relations). Importantly, for any $\mu$-independent choice for $m_{i}, \mathbf{M}$ is independent of $\mu$.

The effect of the color-trivial component of eq. (5.2) (i.e., the contribution from the term in brackets in eq. (5.4)) can be obtained using the results in appendix B and gives rise to a factor $\Pi_{H}$ as in eq. (B.8) with the parameters needed for $K_{H}$ and $\omega_{H}$ at NLL' given in table 2 . We can straightforwardly include the effect of $\Gamma_{c}\left(\alpha_{s}\right) \mathbf{M}\left(m_{i}\right)$ via matrix exponentiation and record the solution as

$$
\mathbf{H}\left(\mu, \mu_{H}\right)=\Pi_{H}\left(\mu, \mu_{H}\right) \boldsymbol{\Pi}_{H}\left(\mu, \mu_{H}\right) \mathbf{H}\left(\mu_{H}\right) \boldsymbol{\Pi}_{H}^{\dagger}\left(\mu, \mu_{H}\right),
$$

where

$$
\boldsymbol{\Pi}_{H}\left(\mu, \mu_{H}\right) \equiv \exp \left\{\mathbf{M} \int_{\alpha_{s}\left(\mu_{H}\right)}^{\alpha_{s}(\mu)} \frac{d \alpha}{\beta[\alpha]} \Gamma_{c}(\alpha)\right\}=\exp \left\{\mathbf{M}\left(\frac{2}{\beta_{0}} \ln \frac{\alpha_{s}\left(\mu_{H}\right)}{\alpha_{s}(\mu)}+\cdots\right)\right\},
$$

where in the second equality we expanded to NLL' accuracy. This matrix exponential can be defined by first constructing the matrix $\mathbf{R}$ of eigenvectors of $\mathbf{M}$ such that $\mathbf{R}^{-1} \mathbf{M R}=\boldsymbol{\Lambda}_{H}$ is the diagonal matrix of eigenvalues of $\mathbf{M}$, and then defining $\exp (\mathbf{M}) \equiv \mathbf{R} \exp \left(\boldsymbol{\Lambda}_{H}\right) \mathbf{R}^{-1}$.

\subsection{Jet functions and unmeasured beam functions}

Since the jet functions can be obtained directly from rescalings of those in ref. [12] as described in section 4.1, the renormalization is similarly related to the results in ref. [12]. For measured (renormalized) jet functions we have

$$
\gamma_{J_{i}}\left(\tau_{a}^{i}, \mu\right)=\left[2 \Gamma_{c}\left(\alpha_{s}\right) C_{i} \frac{2-a}{1-a} \ln \frac{\mu}{p_{T}}+\frac{\alpha_{s}}{\pi} \gamma_{i}\right] \delta\left(\tau_{a}^{i}\right)-2 \Gamma_{c}\left(\alpha_{s}\right) C_{i} \frac{1}{1-a}\left(\frac{1}{\tau_{a}^{i}}\right)_{+},
$$

which is of the general form eq. (B.12) with cusp $\left(\Gamma_{F}\left[\alpha_{s}\right]\right)$ and non-cusp $\left(\gamma_{F}\left[\alpha_{s}\right]\right)$ pieces given in table 2. Here and below, the ' + ' distribution is defined for example in eq. (A.2) of ref. [12].

To RG evolve the jet function, we perform the integral in eq. (B.13) for the case $F=J$. Integrals of this form are most easily performed by convolving the right-hand side against $1=Z^{-1} \otimes Z$ and first performing the convolution of $U_{F}$ with the bare function, i.e., $Z \otimes F$, then expanding in $\epsilon$, and finally performing the $Z^{-1}$ convolution (which just removes the $1 / \epsilon$ poles in a minimal subtraction scheme). For the jet function, we obtain

$$
\begin{aligned}
J^{\text {meas }}\left(\tau_{a}^{i}, \mu\right)= & Z_{J}^{-1}\left(\tau_{a}^{i}, \mu_{J}\right) \otimes\left[J^{\text {meas }}\left(\tau_{a}^{i}\right) \otimes U_{J}\left(\tau_{a}^{i}, \mu, \mu_{J}\right)\right] \\
= & Z_{J}^{-1}\left(\tau_{a}^{i}, \mu_{J}\right) \otimes\left\{U _ { J } ( \tau _ { a } ^ { i } , \mu , \mu _ { J } ) \left(1-\frac{\alpha_{s}\left(\mu_{J}\right)}{2 \pi}\left[-f_{i}(a)\right.\right.\right. \\
& \left.\left.\left.\quad+\left(\frac{1}{\epsilon} \frac{2 C_{i}}{1-a}+\frac{\gamma_{i}}{1-a / 2}\right) \frac{\Gamma(-2 \epsilon /(2-a)) \Gamma\left(-\omega_{J}^{i}\right)}{\Gamma\left(-2 \epsilon /(2-a)-\omega_{J}^{i}\right)}\left(\frac{\mu_{J}}{p_{T}\left(\tau_{a}^{i}\right)^{1 /(2-a)}}\right)^{2 \epsilon}\right]\right)\right\}_{+} \\
= & \left\{U_{J}\left(\tau_{a}^{i}, \mu, \mu_{J}\right)\left(1+f_{J}^{i}\left(\tau_{a}^{i} ; \omega_{J}^{i}, \mu_{J}\right)\right)\right\}_{+},
\end{aligned}
$$


where $f_{J}(\tau, \Omega, \mu)$ is the one loop part of the renormalized jet function after RG evolution,

$$
\begin{aligned}
f_{J}^{i}(\tau, \Omega, \mu)=\frac{\alpha_{s}}{\pi(2-a)}\{ & \frac{2-a}{2} f_{i}(a)+\gamma_{i}\left[H(-1-\Omega)+(2-a) \ln \frac{\mu}{p_{T} \tau^{1 /(2-a)}}\right] \\
& \left.+\frac{C_{i}}{1-a}\left[\left(H(-1-\Omega)+(2-a) \ln \frac{\mu}{p_{T} \tau^{1 /(2-a)}}\right)^{2}-\psi^{(1)}(-\Omega)+\frac{\pi^{2}}{6}\right]\right\},
\end{aligned}
$$

and $H(x)$ is the harmonic number function and $\psi^{(1)}(x)$ is the polygamma function of order 1 and $f_{i}(a)$ is given in eq. (4.9). The natural scale for the jet function suggested by eq. (5.11) is

$$
\mu_{J}^{\text {meas }} \equiv p_{T}\left(\tau_{a}^{i}\right)^{1 /(2-a)} .
$$

From the discussion in section 4.2 and the results of section 4.1, we have for both unmeasured jet functions and unmeasured beam functions the anomalous dimensions

$$
\gamma_{J_{i}}=2 \Gamma_{c}\left(\alpha_{s}\right) C_{i} \ln \frac{\mu}{p_{T} \mathcal{R}}+\frac{\alpha_{s}}{\pi} \gamma_{i}
$$

and

$$
\gamma_{B_{i}}=2 \Gamma_{c}\left(\alpha_{s}\right) C_{i} \ln \frac{\mu}{x_{i} \mathrm{E}_{\mathrm{cm}} e^{-y_{\mathrm{cut}}}}+\frac{\alpha_{s}}{\pi} \gamma_{i}
$$

which have the form of eq. (B.4). We have summarized the cusp and non-cusp parts in table 2 and $\gamma_{i}$ is given in eq. (4.2) for quark and gluon jets. Eqs. (5.13) and (5.14) (together with eq. (4.1)) suggests the canonical scale choices

$$
\mu_{J}^{\text {unmeas }}=p_{T} \mathcal{R} \quad \text { and } \quad \mu_{B}=x_{i} \mathrm{E}_{\mathrm{cm}} e^{-y_{\mathrm{cut}}},
$$

with $x_{i}$ fixed via eq. (3.1).

\subsection{Soft function}

The total measured soft function, which includes both the $\mathbf{S}^{\text {unmeas }}$ and a $S^{\text {meas }}$ contribution for each measured jet as in eq. (4.14), can be evolved by using a multiplicative-type RGE (cf. eq. (B.2)) for $\mathbf{S}^{\text {unmeas }}$ and a convolution-type RGE (cf. eq. (B.10)) for $S^{\text {meas }}$, and each can be evolved from a separate scale (an unmeasured soft scale and a measured soft scale, respectively). This corresponds an early version of "refactorization" originally suggested in ref. [12]. A more complete refactorization procedure was recently introduced in [36] which involves further refactorizing $\mathbf{S}^{\text {unmeas }}$ into a global soft contribution and a soft-collinear contribution, as in eq. (4.31). In this section, we demonstrate how both approaches are achieved so that they can be compared numerically in section 5.5.

\subsubsection{Unmeasured evolution}

The unmeasured component of the soft function $\mathbf{S}^{\text {unmeas }}$ is renormalized much like the hard function $^{5}$

$$
\mathbf{S}^{\text {unmeas, bare }}=\mathbf{Z}_{S}^{\dagger}(\mu) \mathbf{S}^{\text {unmeas }}(\mu) \mathbf{Z}_{S}(\mu)
$$

\footnotetext{
${ }^{5}$ Note that eq. (5.16) takes the form of eq. (5.1) but with $\mathbf{Z}_{H} \leftrightarrow \mathbf{Z}_{S}^{\dagger}$. This gives rise to the RGE eq. (5.17) which is of the form eq. (5.2) but with $\boldsymbol{\Gamma}_{S}^{\text {unmeas }} \leftrightarrow \boldsymbol{\Gamma}_{H}^{\dagger}$. RGE invariance then requires $\boldsymbol{\Gamma}_{H}=-\boldsymbol{\Gamma}_{S}^{\text {unmeas }}+\cdots$ where the ellipses denote color-trivial contributions.
} 
which gives rise to an RGE of the form

$$
\frac{d}{d \ln \mu} \mathbf{S}^{\text {unmeas }}=\mathbf{S}^{\text {unmeas }} \boldsymbol{\Gamma}_{S}^{\text {unmeas }}+\text { h.c. },
$$

with

$$
\begin{aligned}
\Gamma_{S}^{\text {unmeas }} & \equiv \frac{\alpha_{s}}{\pi}\left(\mathbf{S}^{\text {div }}-i \pi \mathbf{T}+\sum_{i=1,2} C_{i} \ln \mathcal{R}\right) \\
& =\frac{\alpha_{s}}{\pi}\left(\Delta \gamma_{s s}\left(m_{i}\right)-\mathbf{M}\left(m_{i}\right)+\sum_{i=1,2} C_{i} \ln \mathcal{R}\right)
\end{aligned}
$$

where $\mathbf{S}^{\mathrm{div}}$ and $\Delta \gamma_{s s}$ are defined in eqs. (4.29) and (4.30), and $\mathbf{M}$ and $\mathbf{T}$ are defined in eqs. (5.5) and (5.6). In eq. (5.18), we have inserted the factor $i \pi \mathbf{T}$ to comply with matrixlevel consistency of the anomalous dimensions, which is consistent with the one loop bare soft function calculation eq. (4.28) since $\mathbf{S}_{0} \mathbf{T}=\mathbf{T}^{\dagger} \mathbf{S}_{0}$.

The solution to this RGE is completely analogous to that of the hard RGE eq. (5.2). The result is

$$
\mathbf{S}^{\text {unmeas }}\left(\mu, \mu_{S}\right)=\Pi_{S}^{\text {unmeas }}\left(\mu, \mu_{S}\right)\left[\boldsymbol{\Pi}_{S}^{\dagger}\left(\mu, \mu_{S}\right) \mathbf{S}^{\text {unmeas }}\left(\mu_{S}\right) \boldsymbol{\Pi}_{S}\left(\mu, \mu_{S}\right)\right]
$$

where $\Pi_{S}^{\text {unmeas }}$ is of the form eq. (B.8) with NLL' parameters given in table 2 and

$$
\boldsymbol{\Pi}_{S}\left(\mu, \mu_{S}\right) \equiv \exp \left\{-\mathbf{M} \int_{\alpha_{s}\left(\mu_{S}\right)}^{\alpha_{s}(\mu)} \frac{d \alpha}{\beta[\alpha]} \Gamma_{c}(\alpha)\right\}=\exp \left\{-\mathbf{M}\left[\frac{2}{\beta_{0}} \ln \frac{\alpha_{s}\left(\mu_{S}\right)}{\alpha_{s}(\mu)}+\cdots\right]\right\}
$$

where in the second equality we expanded to NLL' accuracy. Inspection of the unmeasured soft function eq. (4.28) suggests the canonical unmeasured soft scale choice

$$
\mu_{S}^{\text {unmeas }} \equiv p_{T}^{\text {cut }}
$$

\subsubsection{Measured evolution}

When the jets are measured, RGE takes the form

$$
\frac{d}{d \ln \mu} \mathbf{S}\left(\tau_{a}^{1}, \tau_{a}^{2}, \mu\right)=\int d \tau^{\prime} d \tau^{\prime \prime}\left[\mathbf{S}\left(\tau^{\prime}, \tau^{\prime \prime}, \mu\right) \boldsymbol{\Gamma}_{S}\left(\tau_{a}^{1}-\tau^{\prime}, \tau_{a}^{2}-\tau^{\prime \prime}, \mu\right)+\text { h.c. }\right],
$$

with the soft anomalous dimension given to NLL accuracy by

$$
\boldsymbol{\Gamma}_{S}\left(\tau_{a}^{1}, \tau_{a}^{2}, \mu\right)=\boldsymbol{\Gamma}_{S}^{\mathrm{unmeas}} \delta\left(\tau_{a}^{1}\right) \delta\left(\tau_{a}^{2}\right)+\left[\frac{1}{2} \gamma_{S}^{\text {meas }}\left(\tau_{a}^{1}, \mu\right) \delta\left(\tau_{a}^{2}\right)+(1 \leftrightarrow 2)\right],
$$

where $\gamma^{\text {meas }}$ is given by

$$
\gamma_{S}^{\text {meas }}\left(\tau_{a}^{i}, \mu\right)=-\Gamma_{c}\left(\alpha_{s}\right) C_{i} \frac{1}{1-a}\left\{2 \ln \frac{\mu \mathcal{R}^{1-a}}{p_{T}} \delta\left(\tau_{a}^{i}\right)-2\left(\frac{1}{\tau_{a}^{i}}\right)_{+}\right\}
$$

which has the form of eq. (B.12). The $\tau_{a}$ dependence of measured jets requires the inclusion of the evolution kernels $U_{S}^{i}\left(\tau_{a}, \mu, \mu_{0}\right)$ as in eq. (B.14) with NLL' parameters given in table 2. 
To evaluate the effect of convolving these kernels, we use the same method as in eqs. (5.10) and (5.11). This gives for the RG evolved measured part of the soft function

$$
\begin{aligned}
S^{\text {meas }}\left(\tau_{a}^{i} ; \mu\right)= & Z_{S}^{-1}\left(\tau_{a}^{i}, \mu_{S}\right) \otimes\left[U_{S}^{i}\left(\tau_{a}^{i}, \mu, \mu_{S}\right)(1\right. \\
& +\frac{1}{\epsilon} \frac{\alpha_{s}\left(\mu_{S}\right) C_{i}}{\pi(1-a)} \frac{e^{\gamma_{E} \epsilon}}{\Gamma(1-\epsilon)} \\
& \left.\left.\times \frac{\Gamma(-2 \epsilon) \Gamma\left(-\omega_{S}^{i}\right)}{\Gamma\left(-2 \epsilon-\omega_{S}^{i}\right)}\left(\frac{\mu_{S} \mathcal{R}^{1-a}}{p_{T} \tau_{a}^{i}}\right)^{2 \epsilon}\right)\right]_{+} \\
= & U_{S}^{i}\left(\tau_{a}^{i}, \mu, \mu_{S}\right)\left(1+f_{S}^{i}\left(\tau_{a}^{i} ; \omega_{S}^{i}, \mu_{S}\right)\right), \\
f_{S}^{i}(\tau ; \Omega, \mu) & =\frac{\alpha_{S} C_{i}}{\pi(1-a)}\left[\psi^{(1)}(-\Omega)-\left(H(-1-\Omega)+\ln \frac{\mu \mathcal{R}^{1-a}}{p_{T} \tau}\right)^{2}-\frac{\pi^{2}}{8}\right],
\end{aligned}
$$

which suggests the canonical scale choice

$$
\mu_{S}^{\text {meas }} \equiv \frac{p_{T} \tau_{a}^{i}}{\mathcal{R}^{1-a}}
$$

Taking the scales from which the two measured components and the unmeasured component are evolved from to be $\mu_{S}^{1,2}$ and $\bar{\mu}_{S}$, respectively, we record the final result as

$$
\begin{aligned}
\mathbf{S}\left(\tau_{a}^{1}, \tau_{a}^{2}, \mu, \mu_{S}^{1}, \mu_{S}^{2}, \bar{\mu}_{S}\right)= & U_{S}^{1}\left(\tau_{a}^{1}, \mu, \mu_{S}^{1}\right) U_{S}^{1}\left(\tau_{a}^{2}, \mu, \mu_{S}^{2}\right)\left[1+\left(f_{S}^{1}\left(\tau_{a}^{1} ; \omega_{S}^{1}, \mu_{S}^{1}\right)+f_{S}^{2}\left(\tau_{a}^{2} ; \omega_{S}^{2}, \mu_{S}^{2}\right)\right)\right] \\
& \times \Pi_{S}^{\text {unmeas }}\left(\mu, \bar{\mu}_{S}\right)\left[\boldsymbol{\Pi}_{S}^{\dagger}\left(\mu, \bar{\mu}_{S}\right) \mathbf{S}^{\text {unmeas }}\left(\bar{\mu}_{S}\right) \boldsymbol{\Pi}_{S}\left(\mu, \bar{\mu}_{S}\right)\right] .
\end{aligned}
$$

\subsubsection{Refactorized evolution}

The components of the refactorized $\mathbf{S}^{\text {unmeas }}$ (cf. eq. (4.31)), $\mathbf{s}_{s}$ and $s_{c s}^{k}$ for $k=1,2$ evolve as

$$
\frac{d}{d \ln \mu} \mathbf{s}_{s}(E)=\int d E^{\prime} \mathbf{s}_{s}\left(E^{\prime}\right) \boldsymbol{\Gamma}_{s s}\left(E-E^{\prime}\right)
$$

and

$$
\frac{d}{d \ln \mu} s_{s c}^{k}(E \mathcal{R})=\int d E^{\prime} s_{s c}^{k}\left(E^{\prime} \mathcal{R}\right) \Gamma_{s c}^{k}\left(\left(E-E^{\prime}\right) \mathcal{R}\right),
$$

respectively. The anomalous dimensions take the form eq. (B.12) and satisfy the relations

$$
\frac{1}{2} \int_{0}^{p_{T}^{\text {cut }}} d E \Gamma_{s c}^{k}(E)=-C_{k} \Gamma_{c}\left[\alpha_{s}\right] \ln \frac{\mu}{p_{T}^{\text {cut }} \mathcal{R}}+\gamma_{\text {hemi }}^{k}\left[\alpha_{s}\right],
$$

and

$$
\frac{1}{2} \int_{0}^{p_{T}^{\text {cut }}} d E \boldsymbol{\Gamma}_{s s}(E)=\sum_{i=1,2}\left(C_{i} \Gamma_{c}\left[\alpha_{s}\right] \ln \frac{\mu}{p_{T}^{\text {cut }}}-\gamma_{\text {hemi }}^{i}\left[\alpha_{s}\right]\right)+\frac{\alpha_{s}}{\pi}\left(\Delta \gamma_{s s}\left(m_{i}\right)-\mathbf{M}\left(m_{i}\right)\right),
$$

where we used that to all-orders, the non-cusp part of the anomalous dimension for $\gamma_{s c}$ is the same as that of the hemisphere thrust distribution [36] (of the color-representation of jet $k$ ). At $\mathcal{O}\left(\alpha_{s}\right), \gamma_{\text {hemi }}^{i}=0$. The additional non-cusp parts of eq. (5.32) (which do not appear in the analogous $e^{+} e^{-}$calculation [36]) are needed for this measurement to ensure the consistency of refactorization at $\mathcal{O}\left(\alpha_{s}\right)$,

$$
\frac{1}{2} \int_{0}^{p_{T}^{\text {cut }}} d E\left(\boldsymbol{\Gamma}_{s s}(E)+\sum_{k} \Gamma_{s c}^{k}(E)\right)=\Gamma_{S}^{\text {unmeas }}
$$


To RG evolve the refactorized soft function, we write

$$
\begin{aligned}
\mathbf{S}_{s s}^{(1)} & =\frac{1}{\epsilon}\left(\frac{\mu}{p_{T}^{\text {cut }}}\right)^{2 \epsilon} \mathbf{f}_{s} \\
\sum_{k=1,2} S_{s c}^{k(1)} & =\frac{1}{\epsilon}\left(\frac{\mu}{p_{T}^{\text {cut }} \mathcal{R}}\right)^{2 \epsilon} f_{c}
\end{aligned}
$$

where $f_{s, c}=\sum_{\{i=0,1,2\}} \epsilon^{i-1} f_{s, c}^{i}$ can be read off from the $\mathcal{O}\left(\alpha_{s}\right)$ results eqs. (4.33) and (4.34) and are given by

$$
\begin{array}{ll}
f_{c}^{0}=-2\left(C_{1}+C_{2}\right) & \mathbf{f}_{s}^{0}=-f_{c}^{0} \\
f_{c}^{1}=0 & \mathbf{f}_{s}^{1}=4 \mathbf{S}^{\text {div }} \\
f_{c}^{2}=\frac{\pi^{2}}{6}\left(C_{1}+C_{2}\right) & \mathbf{f}_{s}^{2}=-8 \mathbf{T}_{1} \cdot \mathbf{T}_{2} \ln \left(1+e^{\Delta y}\right) \ln \left(1+e^{-\Delta y}\right)-f_{c}^{2} .
\end{array}
$$

This allows us to write the RG evolved bare functions (using a similar argument as that described above eq. (5.10)) as

$$
\begin{aligned}
& \int_{0}^{p_{T}^{\text {cut }}} d E\left[\mathbf{s}_{s}(E) \otimes U_{s s}\left(E / \mu_{s s}, \mu, \mu_{s s}\right)\right] \otimes_{i=1,2}\left[s_{s c}^{i}(E \mathcal{R}) \otimes U_{s c}^{i}\left(E \mathcal{R} / \mu_{s c}, \mu, \mu_{s c}\right)\right] \\
&= \int_{0}^{p_{T}^{\text {cut }}} d E\left[\mathbf{s}_{s}(E) \otimes_{i=1,2} s_{s c}^{i}(E \mathcal{R})\right] \otimes\left[U_{s s}\left(E / \mu_{s s}, \mu, \mu_{s s}\right) \otimes_{i=1,2} U_{s c}^{i}\left(E \mathcal{R} / \mu_{s c}, \mu, \mu_{s c}\right)\right] \\
&=\int_{0}^{p_{T}^{\text {cut }}} d E\left[1-2 \frac{\Gamma(-2 \epsilon) \Gamma\left(-\Omega_{S}\right)}{\Gamma\left(-2 \epsilon-\Omega_{S}\right)}\left(\frac{\alpha_{s}\left(\mu_{s s}\right)}{4 \pi}\left(\frac{\mu_{s s}}{E}\right)^{2 \epsilon} \mathbf{f}_{s}\right.\right. \\
&\left.\left.+\frac{\alpha_{s}\left(\mu_{s c}\right)}{4 \pi}\left(\frac{\mu_{s c}}{E \mathcal{R}}\right)^{2 \epsilon} f_{c}\right)\right] U_{S}\left(E, \mu, \mu_{s s}, \mu_{s c}\right),
\end{aligned}
$$

where in the 3rd line we truncated the series in parenthesis to $\mathcal{O}\left(\alpha_{s}\right)$ and we defined

$$
\Omega_{S} \equiv \omega_{s s}\left(\mu, \mu_{s s}\right)+\sum_{i=1,2} \omega_{s c}^{i}\left(\mu, \mu_{s c}\right)
$$

and

$$
U_{S}\left(E, \Omega_{S}, \mu_{s s}, \mu_{s c}\right) \equiv\left[U_{s s}\left(E / \mu_{s s}, \mu, \mu_{s s}\right) \otimes_{i=1,2} U_{s c}^{i}\left(E \mathcal{R} / \mu_{s c}, \mu, \mu_{s c}\right)\right]
$$

and used that $U_{S}$ scales as

$$
U_{S} \propto \frac{1}{\Gamma\left(-\Omega_{S}\right)} E^{-1-\Omega_{S}} .
$$

Expanding in $\epsilon$ and dropping the $1 / \epsilon$ poles gives the renormalized, refactorized and RG evolved $\mathbf{S}^{\text {unmeas }}(\mu)$

$$
\mathbf{S}^{\text {unmeas }}(\mu) \rightarrow \mathbf{S}^{\text {unmeas }}\left(\Omega_{S}, \mu_{s s}, \mu_{s c}\right) \int_{0}^{p_{T}^{\text {cut }}} d E U_{S}\left(E, \Omega_{S}, \mu_{s s}, \mu_{s c}\right)
$$


where

$$
\begin{aligned}
\mathbf{S}^{\text {unmeas }}\left(\Omega, \mu_{s c}, \mu_{s s}\right) \equiv \mathbf{S}_{0}+\left\{\mathbf{S}_{0}[\right. & \frac{\alpha_{s}\left(\mu_{s s}\right)}{4 \pi}\left(\frac{1}{2} \mathbf{f}_{s}^{2}+\mathbf{f}_{s}^{1}\left(\ln \frac{\mu_{s s}}{p_{T}^{\text {cut }}}+H(-\Omega)\right)\right. \\
& \left.+\mathbf{f}_{s}^{0}\left(\frac{\pi^{2}}{6}-\psi^{(1)}(1-\Omega)+\left(\ln \frac{\mu_{s s}}{p_{T}^{\text {cut }}}+H(-\Omega)\right)^{2}\right)\right) \\
& +\frac{\alpha_{s}\left(\mu_{s c}\right)}{4 \pi}\left(\frac{1}{2} f_{c}^{2}+f_{c}^{1}\left(\ln \frac{\mu_{s c}}{p_{T}^{\text {cut }} \mathcal{R}}+H(-\Omega)\right)\right. \\
& \left.\left.\left.+f_{c}^{0}\left(\frac{\pi^{2}}{6}-\psi^{(1)}(1-\Omega)+\left(\ln \frac{\mu_{s c}}{p_{T}^{\text {cut }} \mathcal{R}}+H(-\Omega)\right)^{2}\right)\right)\right]+ \text { h.c. }\right\} .
\end{aligned}
$$

We note that when combined into the full cross section in section 5.4, the $\mu$ dependence can be cancelled to all orders between eq. (5.40) and the remainder of the cross section (using consistency and eq. (5.33)) at the expense of running all factorized components from $\mu_{s s}$ to the scale of the component. This means for example that we have

$$
\Omega_{S} \rightarrow \sum_{i=1,2} \omega_{s c}^{i}\left(\mu_{s s}, \mu_{s c}\right) \equiv \omega_{s c} .
$$

This means in particular we can make the replacement

$$
\mathbf{S}^{\text {unmeas }}(\mu) \rightarrow \mathbf{S}^{\text {unmeas }}\left(\omega_{s c}, \mu_{s s}, \mu_{s c}\right) U_{s c}\left(\omega_{s c}, \mu_{s s}, \mu_{s c}\right)
$$

where

$$
U_{s c}\left(\omega_{s c}, \mu_{s s}, \mu_{s c}\right) \equiv \int_{0}^{p_{T}^{\text {cut }}} d E U_{S}\left(E, \omega_{s c}, \mu_{s s}, \mu_{s c}\right)=\frac{e^{K_{s c}+\gamma_{E} \omega_{s c}}}{\Gamma\left(1-\omega_{s c}\right)}\left(\frac{\mu_{s c}}{p_{T}^{\text {cut }} \mathcal{R}}\right)^{\omega_{s c}}
$$

where $K_{s c} \equiv \sum_{i=1,2} K_{s c}\left(\mu_{s s}, \mu_{s c}\right)$. The parameters needed for $K_{s c}$ and $\omega_{s c}$ at NLL' (which can be expanded as in eq. (B.16)) can be read off from eqs. (5.31) and (5.32) and are given in table 2 .

\subsection{Total NLL' resummed cross section}

For the case of unmeasured jets, we can now readily assemble the ingredients in eq. (3.5) to obtain

$$
\begin{aligned}
d \sigma= & \frac{p_{T}}{8 \pi x_{1} x_{2} E_{\mathrm{cm}}^{4}} \frac{1}{N} B\left(x_{1}, \mu_{B}^{1}\right) \bar{B}\left(x_{2}, \mu_{B}^{2}\right) J_{1}\left(\bar{\mu}_{J}^{1}\right) J_{2}\left(\bar{\mu}_{J}^{2}\right) \Pi^{\text {unmeas }}\left(\bar{\mu}_{S}, \bar{\mu}_{J}^{1,2}, \mu_{B}^{1,2}, \mu_{H}\right) \\
& \times \operatorname{Tr}\left\{\mathbf{H}\left(\mu_{H}\right) \boldsymbol{\Pi}^{\dagger}\left(\bar{\mu}_{S}, \mu_{H}\right) \mathbf{S}^{\text {unmeas }}\left(\bar{\mu}_{S}\right) \boldsymbol{\Pi}\left(\bar{\mu}_{S}, \mu_{H}\right)\right\}
\end{aligned}
$$

where here and below we use a bar over a parameter to denote that it is an unmeasured quantity (so for example $\bar{\mu}_{S}$ denotes the unmeasured soft scale while $\mu_{S}$ denotes the measured soft scale), and $x_{1,2}$ are fixed to the values in eq. (3.1). The function $\Pi$ in eq. (5.45) is defined as

$$
\begin{aligned}
\boldsymbol{\Pi}\left(\bar{\mu}_{S}, \mu_{H}\right)=\boldsymbol{\Pi}_{S}\left(\mu, \bar{\mu}_{S}\right) \boldsymbol{\Pi}_{H}\left(\mu, \mu_{H}\right) & =\exp \left\{\mathbf{M} \int_{\alpha_{s}\left(\mu_{H}\right)}^{\alpha_{s}\left(\bar{\mu}_{S}\right)} \frac{d \alpha}{\beta[\alpha]} \Gamma_{c}(\alpha)\right\} \\
& =\exp \left\{\mathbf{M}\left[\frac{2}{\beta_{0}} \ln \frac{\alpha_{s}\left(\mu_{H}\right)}{\alpha_{s}\left(\bar{\mu}_{S}\right)}+\cdots\right]\right\}
\end{aligned}
$$




\begin{tabular}{|c|c|c|c|c|c|}
\hline & $\Gamma_{F}\left[\alpha_{s}\right]$ & $\gamma_{F}\left[\alpha_{s}\right]$ & $j_{F}$ & $m_{F}$ & $\mu_{F}$ \\
\hline$\gamma_{H}$ & $-\Gamma \sum_{i} C_{i}$ & $-\sum_{i} \frac{\alpha_{s}}{\pi} \gamma_{i}$ & 1 & $\prod_{i} m_{i}^{C_{i} / \sum_{j} C_{j}}$ & $m_{i}$ \\
\hline$\gamma_{J_{i}}\left(\tau_{a}^{i}\right)$ & $\Gamma C_{i} \frac{2-a}{1-a}$ & $\frac{\alpha_{s}}{\pi} \gamma_{i}$ & $2-a$ & $p_{T}$ & $p_{T}\left(\tau_{a}^{i}\right)^{1 /(2-a)}$ \\
$\gamma_{S}^{\text {meas }}\left(\tau_{a}^{i}\right)$ & $-\Gamma C_{i} \frac{1}{1-a}$ & 0 & 1 & $p_{T} / \mathcal{R}^{1-a}$ & $p_{T} \tau_{a}^{i} / \mathcal{R}^{1-a}$ \\
\hline$\gamma_{J_{i}}$ & $\Gamma C_{i}$ & $\frac{\alpha_{s}}{\pi} \gamma_{i}$ & 1 & $p_{T} \mathcal{R}$ & $p_{T} \mathcal{R}$ \\
\hline$\gamma_{B_{i}}$ & $\Gamma C_{i}$ & $\frac{\alpha_{s}}{\pi} \gamma_{i}$ & 1 & $x_{i} \mathrm{E}_{\mathrm{cm}} e^{-y_{\mathrm{cut}}}$ & $x_{i} \mathrm{E}_{\mathrm{cm}} e^{-y_{\mathrm{cut}}}$ \\
\hline$\gamma_{S}^{\text {unmeas }}$ & 0 & $\frac{2 \alpha_{s}}{\pi} \Delta \gamma_{s s}\left(m_{i}\right)$ & 1 & - & $p_{T}^{\text {cut }}$ \\
\hline$\gamma_{s s}$ & $\Gamma\left(C_{1}+C_{2}\right)$ & $\frac{2 \alpha_{s}}{\pi}\left(C_{1}+C_{2}\right) \ln \mathcal{R}$ & 1 & & $p_{s s}\left(m_{i}\right)$ \\
\hline$\gamma_{s c}^{i}$ & $-\Gamma C_{i}$ & 0 & 1 & $p_{T}^{\text {cut }}$ & $p_{T}^{\text {cut }} \mathcal{R}$ \\
\hline
\end{tabular}

Table 2. Ingredients for anomalous dimensions of the color-trivial parts components to the factorization formula and the corresponding canonical scale choices $\mu_{F}$, which take the form of eqs. (B.4) and (B.12). The hard and (unmeasured) soft components require an additional color-nontrivial factor derived explicitly in the text. Here, $C_{i}$ is the quadratic Casimir $\left(C_{F}\right.$ or $C_{A}$ for quarks and gluons, respectively), $\gamma_{i}$ is given in eq. (4.2), $\Gamma \equiv \Gamma_{c}\left(\alpha_{s}\right)$ is the cusp (given in eq. (B.20)), $x_{i}$ are the momentum fractions of the partons in the beams (fixed via eq. (3.1)), and $\Delta \gamma_{s s}$ is given in eq. (4.30) (and $m_{i}$ is an arbitrary parameter that cancels both within $\boldsymbol{\Gamma}_{H}$ and within $\boldsymbol{\Gamma}_{S}$ and can for example be chosen based on the partonic channel to coincide with the conventions of ref. [64] as described in the text). For refactorizing the soft function as in [36], the last two rows are used in place of $\gamma_{S}^{\text {unmeas }}$.

with $\boldsymbol{\Pi}_{H}$ and $\boldsymbol{\Pi}_{S}$ defined in eqs. (5.8) and (5.20), respectively, where in the second equality we canceled the $\mu$ dependence (to all orders) and in the third equality we expanded to NLL' accuracy. We also used the definition of the overall multiplicative RG kernel as

$$
\begin{aligned}
\Pi^{\text {unmeas }}\left(\bar{\mu}_{S}, \bar{\mu}_{J}^{1,2}, \mu_{B}^{1,2}, \mu_{H}\right) & \equiv \Pi_{H}\left(\mu, \mu_{H}\right) \Pi_{S}^{\mathrm{unmeas}}\left(\mu, \bar{\mu}_{S}\right) \prod_{i=1,2} \Pi_{B}^{i}\left(\mu, \mu_{B}^{i}\right) \prod_{i=1,2} \bar{\Pi}_{J}^{i}\left(\mu, \bar{\mu}_{J}^{i}\right) \\
& =\prod_{F=H, B_{1}, B_{2}, J_{1}, J_{2}} e^{K_{F}\left(\bar{\mu}_{S}, \mu_{F}\right)}\left(\frac{\mu_{F}}{m_{F}}\right)^{\omega_{F}\left(\bar{\mu}_{S}, \mu_{F}\right)}
\end{aligned}
$$

where $m_{F}, K_{F}^{i}, \omega_{F}^{i}$ for $F=J_{i}, B_{i}, H$ are given to NLL' in eq. (B.16) in terms of the parameters of table 2. To arrive at eq. (5.47), we used the consistency of the anomalous dimensions to explicitly cancel the $\mu$ dependence to all orders. Here and below, we denote unmeasured quantities with bars to distinguish them from the corresponding measured quantities below.

When the angularity of one or more jets is measured, we need to include $S^{\text {meas }}\left(\tau_{a}^{i}\right)$ (and its corresponding anomalous dimension $\gamma_{S}^{\text {meas }}\left(\tau_{a}^{i}\right)$ ) for each measured jet, and we need to replace the unmeasured jet functions $J_{i}$ with measured ones $J\left(\tau_{a}^{i}\right)$ (and replace $\left.\bar{\Pi}_{J}^{i} \rightarrow U_{J}\left(\tau_{a}^{i}\right)\right)$. To perform the convolutions for measured jet functions with the measured part of the soft functions, it is easier to first do the convolutions of the evolution factors with each other, and then convolve the resulting full kernel with the renormalized functions. 
For the case of two measured jets, this yields

$$
\begin{aligned}
& d \sigma\left(\tau_{a}^{1}, \tau_{a}^{2}\right)= \frac{p_{T}}{8 \pi x_{1} x_{2} E_{\mathrm{cm}}^{4}} \frac{1}{N} \\
&\left.\left.\times\left[1+\left(x_{S}^{1}, \mu_{B}^{1}\right) \bar{B}\left(\tau_{2}^{1} ; \omega_{S}^{1}, \mu_{S}^{2}\right)\left[\Pi^{\text {meas }}\left(\tau_{a}^{1,2}, \mu_{S}^{1,2}, \bar{\mu}_{S}, \mu_{J}^{1,2}, \mu_{a}^{1} ; \omega_{S}^{1,2}, \mu_{J}^{1}\right)+(1 \leftrightarrow 2)\right)\right]\right]_{+}\right) \\
& \times \operatorname{Tr}\left\{\mathbf{H}\left(\mu_{H}\right) \boldsymbol{\Pi}^{\dagger}\left(\bar{\mu}_{S}, \mu_{H}\right) \mathbf{S}^{\text {unmeas }}\left(\bar{\mu}_{S}\right) \boldsymbol{\Pi}\left(\bar{\mu}_{S}, \mu_{H}\right)\right\}
\end{aligned}
$$

where $f_{J}^{i}(\tau, \Omega, \mu)$ and $f_{S}^{i}(\tau, \Omega, \mu)$ are given in eqs. (5.11) and (5.26), respectively, and we defined

$$
\begin{aligned}
\Pi^{\text {meas }} & \left(\tau_{a}^{1,2}, \mu_{S}^{1,2}, \bar{\mu}_{S}, \mu_{J}^{1,2}, \mu_{B}^{1,2}, \mu_{H}\right) \\
& \equiv \frac{\Pi^{\text {unmeas }}\left(\bar{\mu}_{S}, \mu_{J}^{1,2}, \mu_{B}^{1,2}, \mu_{H}\right)}{\prod_{i=1,2} \bar{\Pi}_{J}^{i}\left(\mu, \mu_{J}^{i}\right)} \prod_{i=1,2} U_{J}^{i}\left(\tau_{a}^{i}, \mu, \mu_{J}^{i}\right) \otimes U_{S}^{i}\left(\tau_{a}^{i}, \mu, \mu_{S}^{i}\right) \\
& =\Pi^{\text {unmeas }}\left(\bar{\mu}_{S}, \mu_{J}^{1,2}, \mu_{B}^{1,2}, \mu_{H}\right) \prod_{i=1,2} \frac{e^{K_{S}^{i}+\gamma_{E} \omega_{S}^{i}}}{\Gamma\left(-\omega_{S}^{i}\right)}\left(\frac{\mu_{S}^{i}}{m_{S}^{i}}\right)^{\omega_{S}^{i}} \frac{\Theta\left(\tau_{a}^{i}\right)}{\left(\tau_{a}^{i}\right)^{1+\omega_{S}^{i}}},
\end{aligned}
$$

where $\gamma_{E}$ is the Euler constant. The $K_{S}^{i}$ and $\omega_{S}^{i}$ appearing in these eqs. (5.48) and (5.49) are expanded to NLL' in eq. (B.16) in terms of the parameters in table 2 and are evaluated at the scales

$$
\begin{aligned}
\omega_{S}^{i} & \equiv \omega_{S}^{i}\left(\mu_{J}^{i}, \mu_{S}^{i}\right) \\
K_{S}^{i} & \equiv K_{S}^{i}\left(\mu_{J}^{i}, \mu_{S}^{i}\right) .
\end{aligned}
$$

To arrive at eq. (5.49), we used that

$$
\gamma_{J_{i}}\left(\tau_{a}^{i}, \mu\right)+\gamma_{S}^{\text {meas }}\left(\tau_{a}^{i}, \mu\right)-\gamma_{J_{i}}(\mu) \delta\left(\tau_{a}^{i}\right)=0
$$

to explicitly cancel the $\mu$ dependence of the measured jet and soft functions and the subtracted out unmeasured jet functions (evaluated at the measured jet scale $\mu_{J}$ ). In particular, eq. (5.51) implies that

$$
\begin{aligned}
e^{K_{S}^{i}\left(\mu_{J}, \mu_{S}\right)}\left(\frac{\mu_{S}}{m_{S}}\right)^{j S \omega_{S}^{i}\left(\mu_{J}, \mu_{S}\right)}= & e^{K_{J}^{i}\left(\mu, \mu_{J}\right)+K_{S}^{i}\left(\mu, \mu_{S}\right)-\bar{K}_{J}^{i}\left(\mu, \mu_{J}\right)} \\
& \times\left(\frac{\mu_{J}}{m_{J}}\right)^{j_{J} \omega_{J}^{i}\left(\mu, \mu_{J}\right)}\left(\frac{\mu_{S}}{m_{S}}\right)^{j_{S} \omega_{S}^{i}\left(\mu, \mu_{S}\right)}\left(\frac{\mu_{J}}{\bar{m}_{J}}\right)^{-\bar{\omega}_{J}^{i}\left(\mu, \mu_{J}\right)}
\end{aligned}
$$

and that

$$
\omega_{S}^{i}\left(\mu_{J}, \mu_{S}\right)=\omega_{S}^{i}\left(\mu, \mu_{S}\right)+\omega_{J}^{i}\left(\mu, \mu_{J}\right) .
$$

Finally, we note that to refactorize the cross section and resum logarithms of $\mathcal{R}$ as in ref. [36], we simply need to make the replacement eq. (5.43) for both the case of unmeasured and of measured jet formula, eqs. (5.45) and (5.48), respectively, and interpret $\bar{\mu}_{S} \rightarrow \mu_{s s}$. We discuss the numerical impact of this effect in the next section. 


\subsection{A simple example}

We consider the simple partonic channel $q q^{\prime} \rightarrow q q^{\prime}$. Of course to compute a physically observable cross section we will need to sum over all partonic channels, however, this is beyond the scope of this work. Our aim is to consider the scale variation of the cross section and investigate the impact of refactorization of the soft function on the differential cross section. We find the main effect of refactorization is to reduce the normalization of the cross section and to lower the scale uncertainty, which is qualitatively similar to what is found in the study of refactorization in $e^{+} e^{-}$collisions recently completed in ref. [36]. We also study the dependence of the cross section on the parameters $\mathcal{R}, p_{T}^{\text {cut }}$, and $a$, and comment on the physics responsible for this dependence.

From the results of ref. [64] we have the ( $\overline{\mathrm{MS}}$ renormalized) hard function to $\mathcal{O}\left(\alpha_{s}\right)$ in the color basis that corresponds to the t-channel $B \otimes B$ and $\mathbb{1} \otimes \mathbb{1}$ operators,

$$
\mathbf{H}(\mu)=8 g^{4}\left(\mathbf{H}_{0}+\frac{\alpha_{s}}{4 \pi} \mathbf{H}_{1}(\mu)+\mathcal{O}\left(\alpha_{s}^{2}\right)\right)
$$

where

$$
\mathbf{H}_{0}=\frac{s^{2}+u^{2}}{t^{2}}\left(\begin{array}{ll}
1 & 0 \\
0 & 0
\end{array}\right),
$$

and

$$
\begin{aligned}
{\left[\mathbf{H}_{1}(\mu)\right]_{11}=} & \frac{s^{2}+u^{2}}{t^{2}}\left(-4 C_{F} \ln ^{2} \frac{-t}{\mu^{2}}+2 \operatorname{Re}\left[X_{1}(s, t, u)\right] \ln \frac{-t}{\mu^{2}}+2 Y\right) \\
& +\frac{s^{2}}{t^{2}}\left(C_{A}-4 C_{F}\right) \operatorname{Re}[Z(s, t, u)]+\frac{u^{2}}{t^{2}}\left(4 C_{F}-2 C_{A}\right) \operatorname{Re}[Z(u, t, s)] \\
{\left[\mathbf{H}_{1}(\mu)\right]_{21}=} & \frac{s^{2}+u^{2}}{t^{2}} X_{2}(s, t, u) \ln \frac{-t}{\mu^{2}}-\frac{s^{2}}{t^{2}} \frac{C_{F}}{2 C_{A}} Z(s, t, u)+\frac{u^{2}}{t^{2}} \frac{C_{F}}{2 C_{A}} Z(u, t, s) \\
{\left[\mathbf{H}_{1}(\mu)\right]_{12}=} & {\left[\mathbf{H}_{1}(\mu)\right]_{21}^{*} } \\
{\left[\mathbf{H}_{1}(\mu)\right]_{22}=} & 0,
\end{aligned}
$$

where $X_{1,2}, Z$, and $Y$ are defined in eqs. (33)-(36) of [64] and $s, t$, and $u$ are given in terms of the jet rapidities and $p_{T}$ in eq. (3.2).

To use the convention of [64], we set $m_{i}=\sqrt{-t}$ for this channel and have

$$
\mathbf{M}^{\prime}(\sqrt{-t})=\left(\begin{array}{cc}
4 C_{F} \ln \frac{-u}{s}-C_{A} \ln \frac{t u}{s^{2}} & 2 \ln \frac{-u}{s} \\
\frac{C_{F}}{C_{A}} \ln \frac{-u}{s} & 0
\end{array}\right)
$$

and

$$
\mathbf{M}(\sqrt{-t})=\mathbf{M}^{\prime}(\sqrt{-t})+i \pi \mathbf{T}
$$

where

$$
\mathbf{T}=\left(\begin{array}{ll}
-2 / C_{A} & 2 \\
C_{F} / C_{A} & 0
\end{array}\right)
$$


Computing the eigenvalues of $\mathbf{M}$ gives

$$
\begin{aligned}
\lambda_{1,2}^{H}= & -\frac{C_{A}}{2}\left(\ln \frac{u t}{s^{2}}+2 i \pi\right)+2 C_{F}\left(\ln \frac{-u}{s}+i \pi\right) \\
& \pm \sqrt{\frac{C_{A}^{2}}{4}\left(\ln \frac{u t}{s^{2}}+2 i \pi\right)^{2}-2 C_{F} C_{A}\left(\ln \frac{-u}{s}+i \pi\right)\left(\ln \frac{-t}{s}+i \pi\right)},
\end{aligned}
$$

and for the eigenvectors we find

$$
\mathbf{R}=\left(\begin{array}{cc}
\lambda_{1}^{H} & \lambda_{2}^{H} \\
\frac{C_{F}}{C_{A}}\left(\ln \frac{-u}{s}+i \pi\right) & \frac{C_{F}}{C_{A}}\left(\ln \frac{-u}{s}+i \pi\right)
\end{array}\right) .
$$

The $\overline{\mathrm{MS}}$ renormalized soft function for the naive factorization is given by

$$
\begin{aligned}
\mathbf{S}^{\text {unmeas }}(\mu)=\mathbf{S}_{0}+\frac{\alpha_{s}}{\pi}\left\{\mathbf{S}_{0}[\right. & \left(\mathbf{S}^{\text {div }}+2 C_{F} \ln \mathcal{R}\right) \ln \frac{\mu}{p_{T}^{\text {cut }}}-C_{F} \ln ^{2} \mathcal{R} \\
& \left.\left.-\mathbf{T}_{1} \cdot \mathbf{T}_{2} \ln \left(1+e^{\Delta y}\right) \ln \left(1+e^{-\Delta y}\right)\right]+ \text { h.c. }\right\},
\end{aligned}
$$

whereas the refactorized result is obtained with the replacement eq. (5.43). The tree level soft function in this basis is given by

$$
\mathbf{S}_{0}=\left(\begin{array}{cc}
\frac{1}{2} C_{F} C_{A} & 0 \\
0 & C_{A}^{2}
\end{array}\right)
$$

In addition to $S_{0}$ and the matrix component $\mathbf{M}^{\prime}\left(m_{i}\right)$ of $\mathbf{S}^{\text {div }}$ given above, we need the matrix $\mathbf{T}_{1} \cdot \mathbf{T}_{2}$, which for a general $2 \rightarrow 2$ scattering is given by

$$
\mathbf{T}_{1} \cdot \mathbf{T}_{2}=\mathbf{T}_{B} \cdot \mathbf{T}_{\bar{B}}+\frac{1}{2}\left(C_{B}+C_{\bar{B}}-C_{1}-C_{2}\right) .
$$

For $q q \rightarrow q q, C_{i}=C_{F}$ for all $i$ so the $C_{i}$ cancel and we have

$$
\mathbf{T}=\frac{1}{2}\left[2 \mathbf{T}_{B} \cdot \mathbf{T}_{\bar{B}}+2 \mathbf{T}_{1} \cdot \mathbf{T}_{2}\right]=2 \mathbf{T}_{1} \cdot \mathbf{T}_{2} .
$$

To estimate uncertainty from higher orders in perturbation theory, we vary the hard scale $\mu_{H}$ and the unmeasured jet and soft scales, $\bar{\mu}_{J}$ and $\bar{\mu}_{S}$, separately by $\pm 50 \%$ around their central values, which we take to be the canonical scales $\mu_{F}$ given in table 2 . For the refactorized case, we vary the soft scales $\mu_{s s}$ and $\mu_{s c}$ simultaneously. However, to avoid varying the measured jet and soft scales for $\mu_{J, S} \sim \Lambda_{\mathrm{QCD}}$, we vary them around profile functions $[65,66]$. This is done by defining $\mu_{J, S}$ as

$$
\begin{aligned}
& \mu_{S}^{i}\left(\tau_{a}^{i}\right)=\left(1+e_{S} g(\tau)\right) \mu\left(\tau_{a}^{i}\right) \\
& \mu_{J}^{i}\left(\tau_{a}^{i}\right)=\left(1+e_{J} g(\tau)\right)\left(p_{T} \mathcal{R}\right)^{\frac{1-a}{2-a}}\left(\mu\left(\tau_{a}^{i}\right)\right)^{\frac{1}{2-a}}
\end{aligned}
$$

with $e_{J, S} \in(-1 / 2,1 / 2)$. The total uncertainty bands are defined to be the envelope of all of the above variations. 

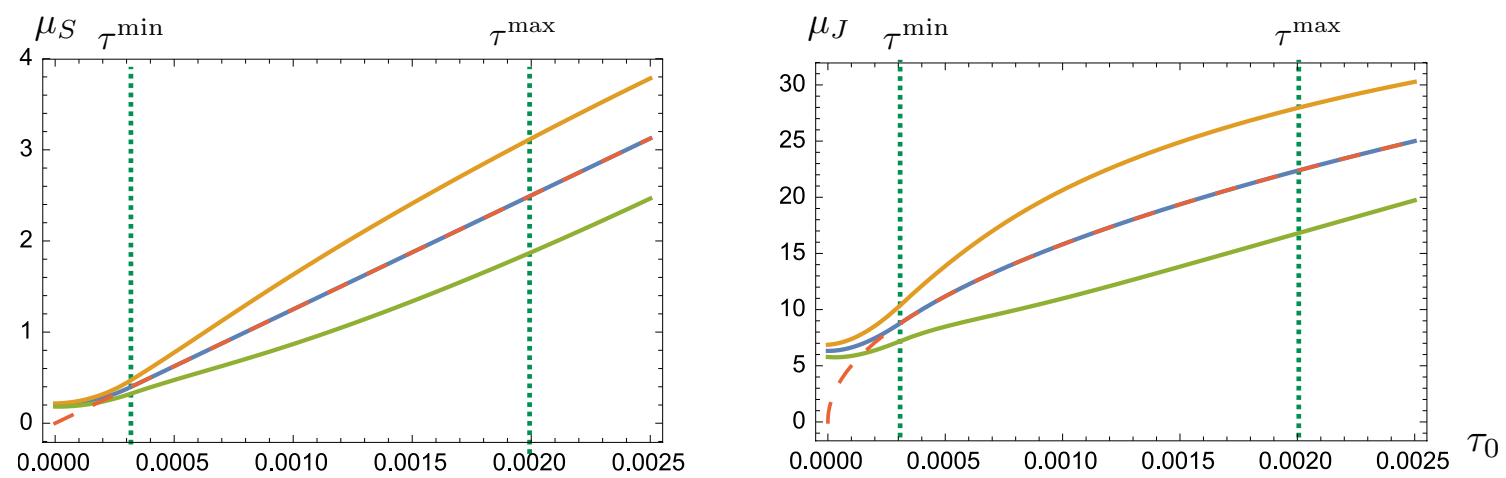

Figure 1. Profile functions for $\mu_{S}$ and $\mu_{J}$. These functions are defined in eq. (5.66) and below.

In terms of the function

$$
\theta_{\epsilon}(x) \equiv \frac{1}{1+\exp (-x / \epsilon)},
$$

which becomes a Heaviside step function in the limit $\epsilon \rightarrow 0$,

$$
\lim _{\epsilon \rightarrow 0} \theta_{\epsilon}(x)=\theta(x)
$$

the function $g(\tau)$ is chosen to be

$$
g(\tau)=\theta_{\epsilon_{1}}\left(\tau-\tau^{\min }\right) \theta_{\epsilon_{2}}\left(\tau^{\max }-\tau\right)
$$

and $\mu(\tau)$ is chosen to be

$$
\mu(\tau)= \begin{cases}\mu_{0}+\alpha \tau^{\beta} \sqrt{-t}, & \tau<\tau^{\min } \\ \frac{p_{T} \tau}{\mathcal{R}^{1-a}}, & \tau>\tau^{\min },\end{cases}
$$

where $\alpha$ and $\beta$ are fixed by the continuity of $\mu(\tau)$ and its first derivative to be

$$
\begin{aligned}
\alpha & =\frac{p_{T}}{\beta\left(\tau^{\min }\right)^{\beta-1} \mathcal{R}^{1-a} \sqrt{-t}} \\
\beta & =\left(1-\frac{\mu_{0} R^{1-a}}{p_{T} \tau^{\text {min }}}\right)^{-1},
\end{aligned}
$$

respectively. The continuity conditions also require that $\beta$ is greater than unity which implies we need $\tau^{\min }>\mu_{0} \mathcal{R}^{1-a} / p_{T}$.

The profile functions for $\mu_{S}$ and $\mu_{J}$, for $a=0$, are shown in figure 1. Eqs. (5.69) and (5.70) together ensure that for sufficiently small $\tau$, the scale choice becomes frozen to be $\mu_{0}$ (and non-perturbative physics dominates), above some scale $\tau^{\min }$ we recover the canonical choices (cf. $m_{J, S}$ of table table 2 ), and above a third scale $\tau^{\max }$ individual $H, J, S$ scale variation begins to dampen (as that should be handled by the traditional $\mu$ variation of fixed-order QCD using a tail-region matching scheme). This is expected to give reasonable scale variation for the range of validity, roughly $\tau^{\min }<\tau<\tau^{\max }$. 
For the sake of illustration, we plot the "normalized cross section" (which neglects the PDFs and effects of the fixed order beam function corrections, the latter of which can be found in [55] following the discussion in section 4.2), defined as

$$
\left.d \tilde{\sigma}\left(\tau_{a}\right) \equiv \frac{B\left(x_{1}, \mu=\mu_{H}\right) \bar{B}\left(x_{2}, \mu=\mu_{H}\right)}{B\left(x_{1}, \mu=\mu_{B}^{1}\right) \bar{B}\left(x_{2}, \mu=\mu_{B}^{2}\right)} \frac{d \sigma\left(\tau_{a}^{1}, \tau_{a}^{2}\right)}{\sigma^{\mathrm{LO}}\left(\mu=\mu_{H}\right)}\right|_{\tau_{a}^{1}=\tau_{a}^{2}=\tau_{a}} .
$$

For the kinematic and algorithm/observable parameters, we choose for a set of default parameters (fixed to these values unless explicitly varying them in the figures)

$$
\begin{aligned}
& \mathrm{E}_{\mathrm{cm}}=10 \mathrm{TeV} \quad y_{1}=1.0 \quad p_{T}=500 \mathrm{GeV} \quad \mathcal{R}=0.6 \\
& a=0 \quad y_{2}=1.4 \quad p_{T}^{\text {cut }}=20 \mathrm{GeV} \quad y_{\text {cut }}=5.0,
\end{aligned}
$$

which corresponds to (via eqs. (3.1) and (3.2))

$$
\begin{aligned}
& t / s=-0.401 \\
& u / s=-0.599 \\
& \sqrt{s} / \mathrm{E}_{\mathrm{cm}}=0.051
\end{aligned}
$$

and for the profile functions parameters, we choose

$$
\begin{aligned}
& \tau^{\min }=2(1-a) \mu_{0} \mathcal{R}^{1-a} / p_{T}=.00032(1-a) \\
& \tau^{\max }=.002 \\
& \frac{\epsilon_{1}}{\tau^{\min }}=\frac{\epsilon_{2}}{\tau^{\max }}=10^{-0.1} \\
& \mu_{0}=200 \mathrm{MeV} \text {. }
\end{aligned}
$$

In figure 2 we show the NLL' calculations for four different values of $\mathcal{R}$, with all other parameters set to their default values in eq. (5.73). In these plots the blue bands are the predictions with a refactorized soft function and the red bands are the predictions without refactorization. In the limit $\mathcal{R} \rightarrow 1$ the scales $\mu_{s s}$ and $\mu_{s c}$ coincide and the two calculations must give the same result, as seen in the figure. For the smallest value of $\mathcal{R}=0.4$, refactorization lowers the normalization of the cross sections by a factor of roughly two, without changing the shape of the distribution or the location of the peak. Refactorization gives a small reduction in the scale uncertainty for $\mathcal{R}<1$. Note that as $\mathcal{R}$ decreases the peak in the $\tau_{0}$ distribution shifts to smaller values of $\tau_{0}$ because the jets are narrower.

Figure 3 shows the refactorized NLL' resummed cross section for three different values of $p_{T}^{\text {cut }}$ with all other parameters set to their defaults in eq. (5.73). Interestingly the shape of the distribution and the location of the peak in the cross section are completely independent of $p_{T}^{\text {cut }}$, only the normalization of the cross section is affected. As expected, the cross section is larger for larger values of $p_{T}^{\text {cut }}$. As discussed in the Introduction, the NGLs, which are of the form $\alpha_{s}^{n} \ln ^{n}\left(p_{T}^{\text {cut }} \mathcal{R}^{2} / p_{T}^{J} \tau_{a}\right)$, for $n \geq 2$, combine $p_{T}^{\text {cut }}$ and $\tau_{a}$ in a nontrivial way. It is possible that when the NGLs are included in the calulcation, the location of the peak of the $\tau_{a}$ distribution may no longer be $p_{T}^{\text {cut }}$ independent. Therefore, the dependence of the peak on $p_{T}^{\text {cut }}$ might be an observable that is sensitive to the NGLs.

Figure 4 shows the refactorized NLL' resummed cross section for four different values of $a$ with other parameters set to the default values. As $a$ is made large and negative, the contribution to the angularity from particles collinear to the jet axis is suppressed by large 

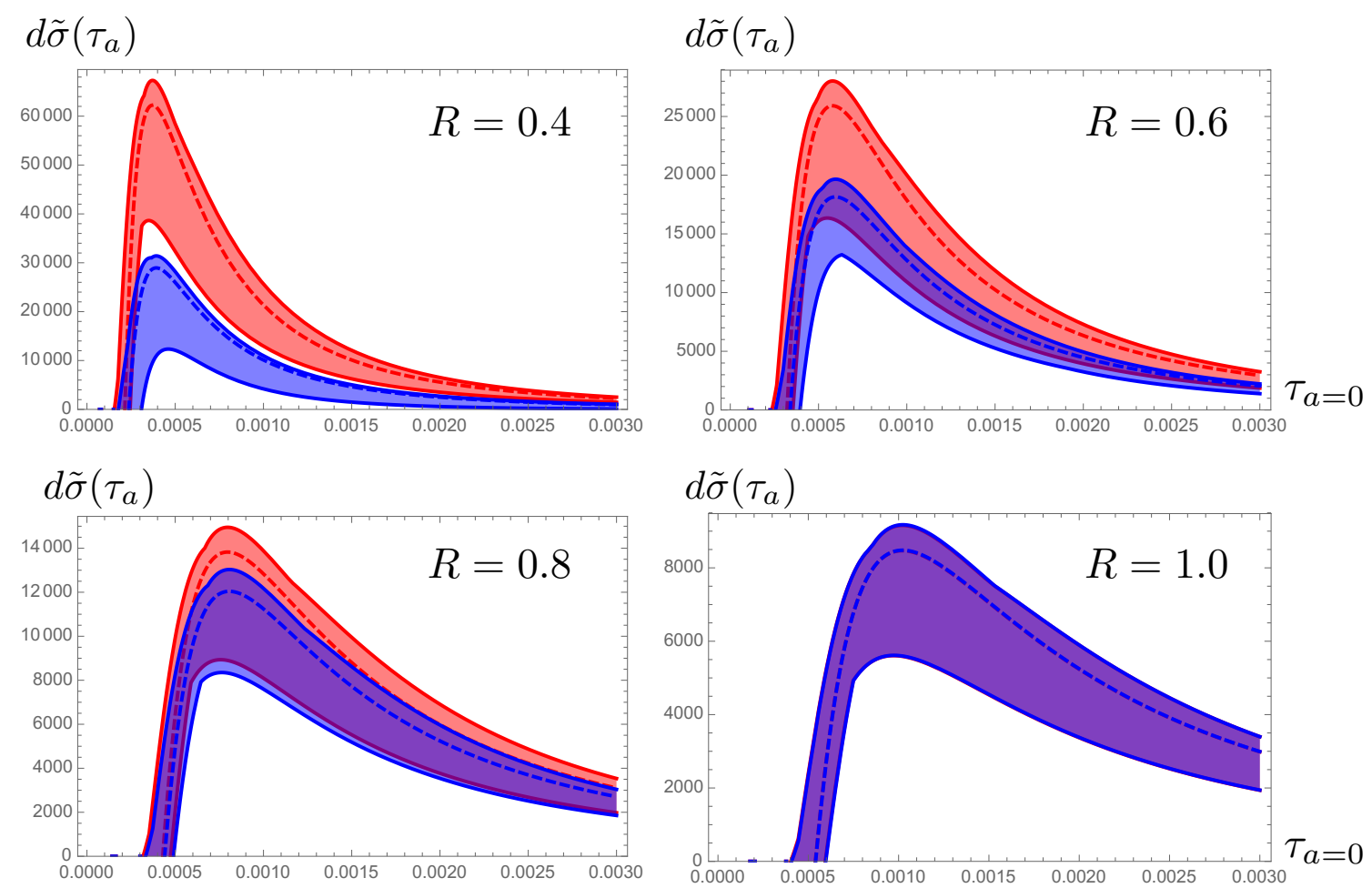

Figure 2. Differential cross section for four different values of $R$ with soft function refactorized (blue) and without (red). Central values are dotted lines and band includes scale variation.

powers of the angle with the jet axis. Correspondingly the distribution is peaked at smaller values of $\tau_{a}$, a behavior also seen in calculations of jet angularities in $e^{+} e^{-}$collisions [12]. It is important for obtaining sensible scale variation for all values of $a$ that the parameter $\tau^{\text {min }}$ defined in eq. (5.75) is proportional $(1-a)$. Both perturbative and power corrections grow with $1 /(1-a)$ and factorization breaks down completely for $a=1$ in $\operatorname{SCET}_{\mathrm{I}}$ (although an SCET $_{\text {II }}$ approach can be used for $\left.a=1[67,68]\right)$. Thus, one expects increasing uncertainty as $a \rightarrow 1$ from below, and we see from figure 3 that the uncertainties in the predictions are substantially larger for $a=0.5$ than for $a \leq 0$.

\section{Conclusion}

In this work, we presented the factorization formulae valid for jet production in hadron colliders with rapidity cuts about the beams, an out-of-jet $p_{T}^{\text {cut }}$ veto, and the jets identified with either a $k_{T}$-type (including $k_{T}, C / A$, and anti- $k_{T}$ ) or cone-type algorithm. We considered the cases that the jets can either be identified but otherwise unprobed ("unmeasured" jets) or are further probed with angularities ("measured" jets). The ingredients of these formulae involved jet functions, unmeasured beam functions, and an observable dependent soft function. This soft function was further written in terms of a universal piece, $\mathbf{S}^{\text {unmeas }}$, which encodes the out-of-jet energy veto $p_{T}^{\text {cut }}$ and angularity independent (but color and direction dependent) pieces. 


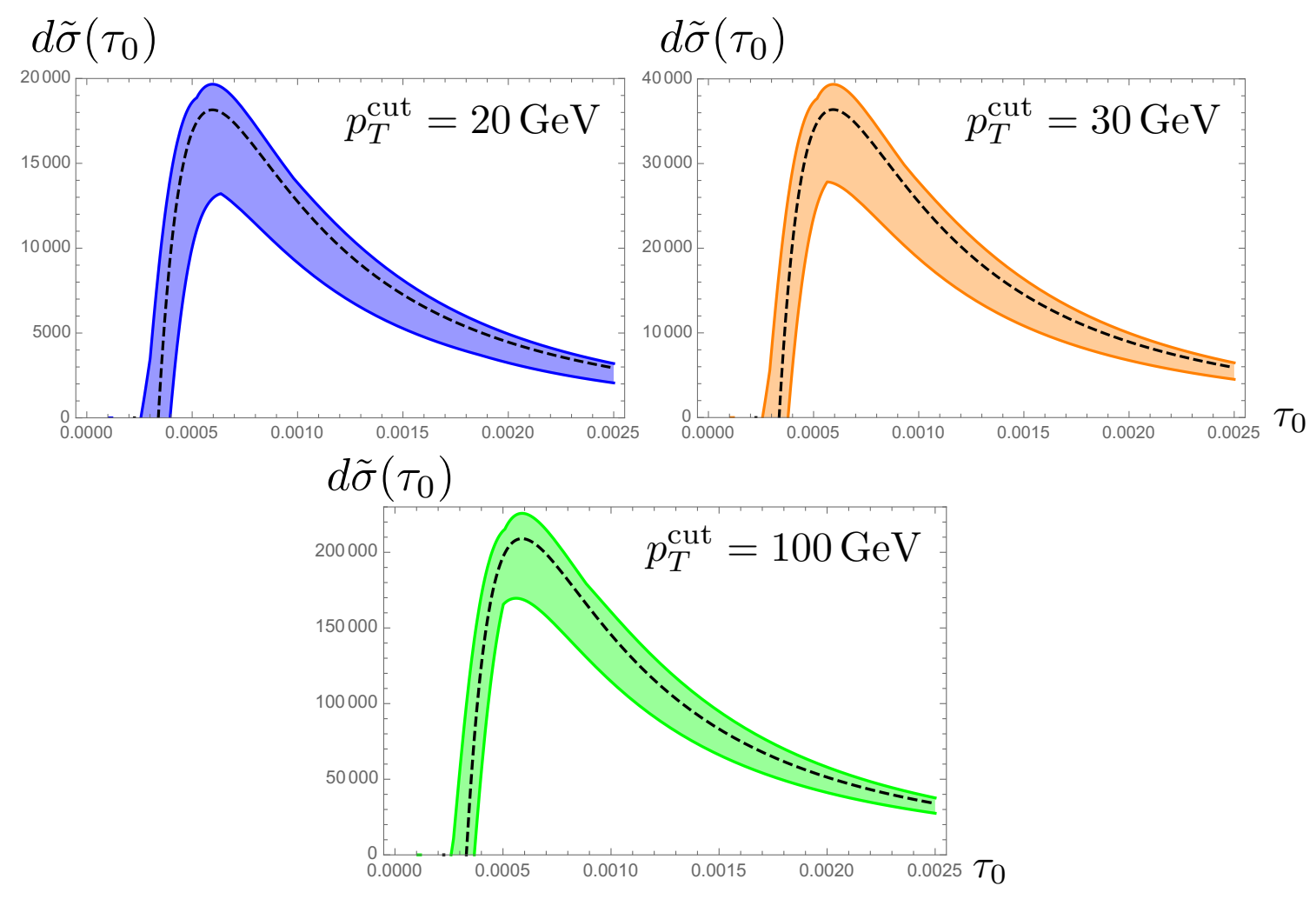

Figure 3. Differential cross sections for three different values of $p_{T}^{\text {cut }}$.

We were able to relate all of the ingredients of the factorization formula except for $\mathbf{S}^{\text {unmeas }}$ to analogous quantities that have previously been calculated in the context of $e^{+} e^{-}$collisions to NLL' accuracy. $\mathbf{S}^{\text {unmeas }}$ was explicitly computed for the case of dijet production (for which all Wilson lines are coplanar) in terms of color operators $\mathbf{T}_{i} \cdot \mathbf{T}_{j}$ that encode the color correlations at this order. We in turn explicitly presented results for these color operators (which become matrices in color space) for the $q q^{\prime} \rightarrow q q^{\prime}$ channel, and plotted the corresponding distribution for the illustrative example where both jets are measured with $\tau_{a}$ for $a=0$ in the $\tau_{a}^{1}=\tau_{a}^{2}$ bin. We also generalized the refactorization of ref. [36] to include color-mixing effects and found that, as was already seen in $e^{+} e^{-}$, the normalization of the cross section and the corresponding scale uncertainty were reduced. Using the results of ref. [36], our results can now be straightforwardly extended to NNLL for any combination of measured (at least for $a=0$ ) and unmeasured jets. The non-global logarithms which we do not include and would appear in a fixed order calculation of the soft function beginning at $\mathcal{O}\left(\alpha_{s}^{2}\right)$ have arguments of order $p_{T}^{\text {cut }} \mathcal{R}^{2} / p_{T}^{J} \tau_{a}$ which for the peak region of the distribution (where we trust our calculation) is $\mathcal{O}(1)$ to within a decade.

Armed with this foundation, we can now (after including all the partonic channels) make meaningful comparisons with Monte Carlo event generators and directly with data. It will be of particular interest to study the sensitivity of the proposed, factorized cross section to effects like multiple parton interactions. Other observables that are sensitive to radiation near the beam pipes like beam thrust [69] have been noted to receive $\mathcal{O}(1)$ 

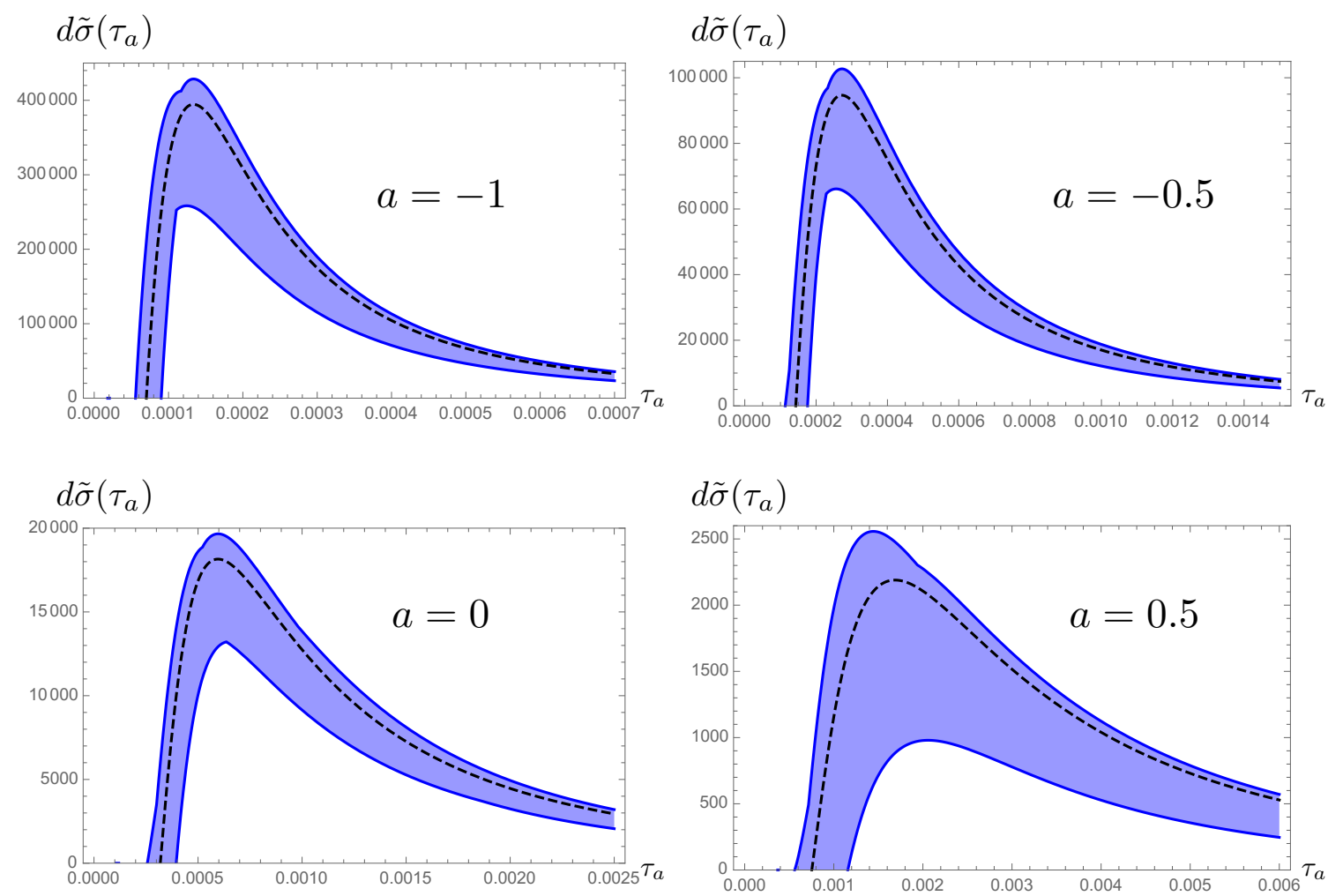

Figure 4. Differential cross sections for four different values of $a$.

corrections from these effects. We expect that our observables will be less sensitive to this effect because the jets are isolated and the unmeasured beam functions should not be sensitive to radiation near the beam pipe. We also hope to be able to incorporate other effects with the recent developments for NGLs as discussed in the Introduction. In addition, the authors, together with other collaborators [70], are actively involved in extending the results of this paper to cross sections for jets in which there is an identified heavy hadron. The work of refs. [55, 57, 71-73] shows that these cross sections can be calculated by replacing the jet function for the jet with the identified hadron with so-called fragmenting jet functions. These are related to the well-known fragmentation functions by a matching calculation at the jet energy scale. These calculations will be applied to the production of jets with open heavy flavor and heavy quarkonia, especially $J / \psi$ and $\Upsilon$. The cross sections will take essentially the same form as the cross sections in this paper, with an additional convolution of the cross section with the heavy quark or quarkonium fragmentation as well as a modified $f_{J}$ factor that depends on the matching coefficients in the fragmenting jet function. We expect to compare these predictions to Monte Carlo event generators and LHC measurements [70]. 


\section{Acknowledgments}

We would like to thank Christopher Lee, Daekyoung Kang, and Wouter Waalewijn for helpful discussions, and Christopher Lee for reviewing this manuscript. AH was supported by a Director's Fellowship from the LANL/LDRD program and the DOE Office of Science under Contract DE-AC52-06NA25396. TM and YM are supported in part by the Director, Office of Science, Office of Nuclear Physics, of the U.S. Department of Energy under grant numbers DE-FG02-05ER41368. TM and YM also acknowledge the hospitality of the theory groups at Brookhaven National Laboratory, Los Alamos National Laboratory, Duke-Kunshan University, and UC-Irvine for their hospitality during the completion of this work.

\section{A Calculations of soft function components}

In this appendix, we calculate the various components needed for $S^{\text {unmeas }}$. As explained in the main body of the text, we only calculate combinations of terms that explicitly remove radiation out of the beams, i.e., with $y>y_{\text {cut }}$ or $y<-y_{\text {cut }}$. We use the definitions $c_{J} \equiv \vec{n}_{J} \cdot \vec{n}_{B}, s_{J} \equiv\left(1-c_{J}^{2}\right)^{1 / 2}, c_{i} \equiv \cos \theta_{i}$, and $s_{i} \equiv \sin \theta_{i}$. All the expressions are special cases of the general form eq. (4.21) in the planar limit, given by the substitution in eq. (4.22). For subtraction terms $S_{i j}^{k}$ defined in eq. (4.19) there is an additional factor of $-\Theta_{\mathcal{R}}^{k}$ given in eq. (4.17).

\section{A.1 Beam-beam interference terms}

We first calculate the beam-beam interference with the gluon out of the beams

$$
\begin{aligned}
\mathcal{I}_{B \bar{B}}^{\text {out }} & \equiv \mathcal{I}_{B \bar{B}}^{\text {incl }}+\mathcal{I}_{B \bar{B}}^{B}+\mathcal{I}_{B \bar{B}}^{\bar{B}} \\
& =\frac{e^{\gamma_{E} \epsilon}}{\sqrt{\pi} \Gamma(1 / 2-\epsilon)} \int_{0}^{\pi} d \theta_{1} \sin \theta_{1} \frac{1}{1-c_{1}} \frac{1}{1+c_{1}} \int_{0}^{\pi} d \theta_{2} \sin ^{-2 \epsilon} \theta_{2} \\
& =\frac{e^{\gamma_{E} \epsilon}}{\Gamma(1-\epsilon)} \int_{-\tanh y_{\text {cut }}}^{\tanh y_{\text {cut }}} \frac{d c_{1}}{1-c_{1}^{2}} \\
& =\ln \frac{1+\tanh y_{\text {cut }}}{1-\tanh y_{\text {cut }}} \\
& =2 y_{\text {cut }} .
\end{aligned}
$$

The region that must be added to remove radiation in the jets goes as $\mathcal{R}^{2}$ and so is power suppressed for small jets, but we record it here for completeness. In a frame where the jet is perpendicular to the beam,

$$
I_{B \bar{B}}^{J}=\frac{e^{\gamma_{E} \epsilon}}{\sqrt{\pi} \Gamma(1 / 2-\epsilon)} \int_{0}^{R} d \theta_{1} \sin ^{1-2 \epsilon} \theta_{1} \int_{0}^{\pi} d \theta_{2} \sin ^{-2 \epsilon} \theta_{2}\left[1-\left(s_{1} c_{2}\right)^{2}\right]^{-1+\epsilon} .
$$

In this frame $\left(\theta_{J}=\pi / 2\right)$, we can make the substitution $R \rightarrow \mathcal{R} \sin \pi / 2=\mathcal{R}$ to get a frame invariant result. This gives

$$
\mathcal{I}_{B \bar{B}}^{J}=\frac{1}{2} \ln \left(1-\mathcal{R}^{2}\right)-\epsilon\left(\frac{\pi^{2}}{12}-\frac{1}{2} \operatorname{Li}_{2}\left(1-\mathcal{R}^{2}\right)\right)=\mathcal{O}\left(\mathcal{R}^{2}\right) .
$$




\section{A.2 Beam-jet interference terms}

The beam-jet interference term with the gluon out of both beams is simplest to compute in the polar coordinates about the beam axis. Defining $\cos \theta_{c} \equiv t_{c} \equiv \tanh y_{\text {cut }}$, it can be written as

$$
\begin{aligned}
\mathcal{I}_{B J}^{\text {out }} & \equiv \mathcal{I}_{B J}^{\text {incl }}+\mathcal{I}_{B J}^{B}+\mathcal{I}_{B J}^{\bar{B}} \\
& =\frac{\left(1-c_{J}\right) e^{\gamma_{E} \epsilon}}{2 \sqrt{\pi} \Gamma(1 / 2-\epsilon)} \int_{\theta_{c}}^{\pi-\theta_{c}} d \theta_{1} \sin \theta_{1} \int_{0}^{\pi} d \theta_{2} \sin ^{-2 \epsilon} \theta_{2} \frac{1}{1-c_{1}} \frac{1}{1-c_{J} c_{1}-s_{J} s_{1} c_{2}} \\
& =\frac{e^{\gamma_{E} \epsilon}}{2} \int_{-t_{c}}^{t_{c}} \frac{d c_{1}}{1-c_{1}^{2}} \frac{1-c_{1}}{1-c_{1} c_{J}}{ }_{2} \tilde{F}_{1}(1 / 2,1 ; 1-\epsilon ; z),
\end{aligned}
$$

where $z=\left(1-c_{1}^{2}\right)\left(1-c_{J}^{2}\right) /\left(1-c_{1} c_{J}\right)^{2}$. We can proceed by extracting the $c_{J}=c_{1}$ singular via the identity

$$
\begin{aligned}
{ }_{2} \tilde{F}_{1}\left(\frac{1}{2}, 1 ; 1-\epsilon ; z\right)=\frac{\sqrt{\pi}}{\Gamma(1 / 2-\epsilon) \cos \pi \epsilon} & {\left[z^{\epsilon}\left(\frac{1-c_{1} c_{J}}{\left|c_{1}-c_{J}\right|}\right)^{1+2 \epsilon}\right.} \\
& \left.+\frac{\epsilon \sqrt{\pi}}{\Gamma(1-\epsilon)}{ }_{2} \tilde{F}_{1}\left(\frac{3}{2}, 1 ; \frac{3}{2}+\epsilon ; 1-z\right)\right] .
\end{aligned}
$$

The singularities are regulated by the $\left|c_{1}-c_{J}\right|^{-1-2 \epsilon}$ in the first term in brackets on the right hand side of eq. (A.5) (and the second term is finite and $\mathcal{O}(\epsilon)$ ). After adding and subtracting the rest of the functional dependence on $c_{1}, f\left(c_{1}\right)$, at the point $c_{1}=c_{J}$ (so that $\left|c_{1}-c_{J}\right|^{-1-2 \epsilon}\left(f\left(c_{1}\right)-f\left(c_{J}\right)\right)$ can safely be expanded in $\left.\epsilon\right)$ and performing some algebra, we arrive at the result

$$
\begin{aligned}
\mathcal{I}_{B J}^{\text {out }}=\frac{e^{\gamma_{E} \epsilon}}{\Gamma(1-\epsilon)}\left\{-\frac{1}{2 \epsilon}+\frac{1}{2}\left[\ln \left(e^{2\left(y_{\mathrm{cut}}-y_{J}\right)}-1\right)+\ln \left(1-e^{-2\left(y_{\mathrm{cut}}+y_{J}\right.}\right)\right]\right. \\
\left.-\epsilon\left[\frac{1}{2} \ln ^{2}\left(1-e^{-2\left(y_{\mathrm{cut}}-y_{J}\right)}\right)+\operatorname{Li}_{2}\left(e^{-2\left(y_{\mathrm{cut}}-y_{J}\right)}\right)+\frac{1}{2} \ln ^{2}\left(1-e^{-2\left(y_{\mathrm{cut}}+y_{J}\right)}\right)\right]\right\} \\
=\frac{e^{\gamma_{E} \epsilon}}{\Gamma(1-\epsilon)}\left[-\frac{1}{2 \epsilon}+y_{\mathrm{cut}}-y_{J}+\mathcal{O}\left(e^{-y_{\mathrm{cut}}}\right)\right] .
\end{aligned}
$$

For the jet region subtraction term $S_{J B}^{J}$, in coordinates about the jet axis, we have

$$
\begin{aligned}
\mathcal{I}_{B J}^{J}= & \frac{\left(1-c_{J}\right) e^{\gamma_{E} \epsilon}}{2 \sqrt{\pi} \Gamma(1 / 2-\epsilon)} \int_{0}^{R} d \theta_{1} \sin ^{1-2 \epsilon} \theta_{1} \int_{0}^{\pi} d \theta_{2} \sin ^{-2 \epsilon} \theta_{2} \\
& \times \frac{1}{1-c_{1}} \frac{1}{1-c_{J} c_{1}-s_{J} s_{1} c_{2}}\left[1-\left(c_{J} c_{1}+s_{J} s_{1} c_{2}\right)^{2}\right]^{\epsilon} \\
= & \frac{\left(1-c_{J}\right) e^{\gamma_{E} \epsilon}}{2 \sqrt{\pi} \Gamma(1 / 2-\epsilon)} \int_{\cos R}^{1} d c_{1}\left(1-c_{1}\right)^{-1-\epsilon} f\left(c_{1}\right)
\end{aligned}
$$

where we defined

$$
f(c)=(1+c)^{-\epsilon} \int_{0}^{\pi} d \theta_{2} \sin ^{-2 \epsilon} \theta_{2} \frac{\left[1-\left(c_{J} c+s_{J}\left(1-c^{2}\right)^{1 / 2} c_{2}\right)^{2}\right]^{\epsilon}}{1-c_{J} c-s_{J}\left(1-c^{2}\right)^{1 / 2} c_{2}} .
$$


Up to corrections that scale as $\mathcal{O}\left(\mathcal{R}^{2}\right)$, we can set $f(c)=f(1)$ which is just

$$
f(1)=\frac{2^{-\epsilon}}{1-c_{J}} s_{J}^{2 \epsilon} \frac{\sqrt{\pi} \Gamma(1 / 2-\epsilon)}{\Gamma(1-\epsilon)} .
$$

Using the substitution eq. (2.6), we find

$$
\mathcal{I}_{B J}^{J}=\frac{e^{\gamma_{E} \epsilon}}{\Gamma(1-\epsilon)} \frac{1}{2 \epsilon} \mathcal{R}^{-2 \epsilon}+\mathcal{O}\left(\mathcal{R}^{2}\right) .
$$

\section{A.3 Jet-jet interference terms}

For the jet-jet interference terms, we work in coordinates about the jet axes in the frame where they are back-to-back, and then convert to lab frame variables. For the term with the gluon allowed anywhere, labeling the jets as 1 and 2, we have in the frame of back-toback jets,

$$
\begin{aligned}
\mathcal{I}_{B J}^{J}= & \frac{e^{\gamma_{E} \epsilon}}{\sqrt{\pi} \Gamma(1 / 2-\epsilon)} \int_{0}^{R} d \theta_{1} \sin ^{1-2 \epsilon} \theta_{1} \int_{0}^{\pi} d \theta_{2} \sin ^{-2 \epsilon} \theta_{2} \\
& \times \frac{1}{1-c_{1}} \frac{1}{1+c_{1}}\left[1-\left(c_{J} c_{1}+s_{J} s_{1} c_{2}\right)^{2}\right]^{\epsilon} \\
= & \frac{e^{\gamma_{E} \epsilon}}{\sqrt{\pi} \Gamma(1 / 2-\epsilon)} 2 \int_{0}^{1} d c_{1}\left(1-c_{1}\right)^{-1-\epsilon} g\left(c_{1}\right),
\end{aligned}
$$

where we defined

$$
g(c)=(1+c)^{-1-\epsilon} \int_{0}^{\pi} d \theta_{2} \sin ^{-2 \epsilon} \theta_{2}\left[1-\left(c_{J} c+s_{J}\left(1-c^{2}\right)^{1 / 2} c_{2}\right)^{2}\right]^{\epsilon} .
$$

As before, we can add and subtract $g(1)$, with

$$
g(1)=\frac{2^{-1-\epsilon}}{1-c_{J}} s_{J}^{2 \epsilon} \frac{\sqrt{\pi} \Gamma(1 / 2-\epsilon)}{\Gamma(1-\epsilon)},
$$

and expand the part of the integrand with $(1-u)^{-1-\epsilon}(f(u)-f(1))$ in $\epsilon$. To evaluate the result, note that

$$
\begin{aligned}
h\left(c_{J}, c_{1}\right) & \equiv \frac{1}{\pi} \int_{0}^{\pi} d \theta \ln \frac{1-\left(c_{1} c_{J}+\left(1-c_{1}^{2}\right)^{1 / 2}\left(1-c_{J}^{2}\right)^{1 / 2} \cos \theta\right)^{2}}{1-c_{J}^{2}} \\
& = \begin{cases}\ln \left[\frac{1-c_{1}^{2}}{1-c_{J}^{2}}\left(\frac{1+\left|c_{J}\right|}{2}\right)^{2}\right] & \text { for }\left|c_{1}\right|<\left|c_{J}\right| \\
2 \ln \frac{1+\left|c_{1}\right|}{2} & \text { for }\left|c_{1}\right|>\left|c_{J}\right|\end{cases}
\end{aligned}
$$

and that

$$
\int_{0}^{1} \frac{d c_{1}}{1-c_{1}^{2}} f\left(c_{J}, c_{1}\right)=-\frac{\pi^{2}}{6}+\frac{1}{2} \ln ^{2} \frac{1-c_{J}}{1+c_{J}}
$$

to finally obtain

$$
\mathcal{I}_{12}^{\text {incl }}=\frac{e^{\gamma_{E} \epsilon}}{\Gamma(1-\epsilon)}\left(\frac{1-\cos ^{2} \theta_{J}}{4}\right)^{\epsilon}\left[-\frac{1}{\epsilon}+\frac{\epsilon}{2} \ln ^{2} \frac{1-c_{J}}{1+c_{J}}\right] .
$$


Noting that $c_{J} \equiv \cos \theta_{J}$ in the back-to-back frame is related to the jet rapidities in the lab frame via $\cos \theta_{J}=\tanh \Delta y / 2$ (cf. eq. (4.24)), we find

$$
\begin{aligned}
\mathcal{I}_{12}^{\text {incl }} & =-\frac{e^{\gamma_{E} \epsilon}}{\Gamma(1-\epsilon)}(2 \cosh (\Delta y / 2))^{-2 \epsilon}\left[\frac{1}{\epsilon}-\frac{\epsilon}{2}(\Delta y)^{2}\right] \\
& =-(2 \cosh (\Delta y / 2))^{-2 \epsilon}\left[\frac{1}{\epsilon}-\frac{\epsilon}{2}(\Delta y)^{2}-\frac{\pi^{2}}{12}\right] .
\end{aligned}
$$

For the jet region subtraction terms, we have

$$
\mathcal{I}_{12}^{1}=\frac{e^{\gamma_{E} \epsilon}}{\sqrt{\pi} \Gamma(1 / 2-\epsilon)} 2 \int_{\cos R}^{1} d c_{1}\left(1-c_{1}\right)^{-1-\epsilon} g\left(c_{1}\right),
$$

which now involves the integral of $h\left(c_{J}, c_{1}\right)$ (cf. eq. (A.14)) over the range $c_{1} \in(\cos R, 1)$ with $c_{J}<\cos R$ (so only the case $\left|c_{1}\right|>\left|c_{J}\right|$ is needed). After some algebra and using the substitution $\tan R / 2 \rightarrow \mathcal{R} /(2 \cosh \Delta y / 2)$, we arrive at the result

$$
\mathcal{I}_{12}^{1}=\frac{e^{\gamma_{E} \epsilon}}{\Gamma(1-\epsilon)} \frac{1}{2 \epsilon} \mathcal{R}^{-2 \epsilon}
$$

\section{B Review of renormalization and RG evolution}

In this appendix we review renormalization and $\mathrm{RG}$ evolution for multiplicatively renormalized functions that are trivial in color-space (namely, the unmeasured jet and beam functions) and for functions of $\tau_{a}$ which renormalize and evolve via a convolution (such as measured jet functions and the measured part of the soft function). The RGE for the non-trivial color-space matrix components of the hard and (unmeasured) soft functions is derived explicitly in section 5.1 and section 5.3, respectively.

Renormalization of the multiplicative-type functions which are trivial in color-space takes the form

$$
F^{\text {bare }}=Z_{F}(\mu) F(\mu)
$$

The independence of the left-hand side on $\mu$ gives rise the RG evolution equation,

$$
\mu \frac{d}{d \mu} F(\mu)=\gamma_{F}(\mu) F(\mu),
$$

where the anomalous dimension $\gamma_{F}$ is defined as

$$
\gamma_{F}(\mu)=-\frac{1}{Z_{F}(\mu)} \mu \frac{d}{d \mu} Z_{F}(\mu),
$$

and to all orders in $\alpha$ takes the form,

$$
\gamma_{F}(\mu)=\Gamma_{F}[\alpha] \ln \frac{\mu^{2}}{m_{F}^{2}}+\gamma_{F}[\alpha]
$$

where $\Gamma_{F}[\alpha]$ and $\gamma_{F}[\alpha]$ have the expansions

$$
\Gamma_{F}\left[\alpha_{s}\right]=\left(\frac{\alpha_{s}}{4 \pi}\right) \Gamma_{F}^{0}+\left(\frac{\alpha_{s}}{4 \pi}\right)^{2} \Gamma_{F}^{1}+\cdots
$$


and

$$
\gamma_{F}\left[\alpha_{s}\right]=\left(\frac{\alpha_{s}}{4 \pi}\right) \gamma_{F}^{0}+\left(\frac{\alpha_{s}}{4 \pi}\right)^{2} \gamma_{F}^{1}+\cdots
$$

The RGE eq. (B.2) has the solution

$$
F(\mu)=\Pi_{F}\left(\mu, \mu_{0}\right) F\left(\mu_{0}\right),
$$

where the evolution kernel $\Pi_{F}$ is given by

$$
\Pi_{F}\left(\mu, \mu_{0}\right)=e^{K_{F}\left(\mu, \mu_{0}\right)}\left(\frac{\mu_{0}}{m_{F}}\right)^{\omega_{F}\left(\mu, \mu_{0}\right)},
$$

where $K_{F}\left(\mu, \mu_{0}\right)$ and $\omega_{F}\left(\mu, \mu_{0}\right)$ will be defined below in eq. (B.15).

Renormalization of functions which depend on the jet shape, $\tau_{a}$, takes the form of a convolution,

$$
F^{\text {bare }}\left(\tau_{a}\right)=\int d \tau_{a}^{\prime} Z_{F}\left(\tau_{a}-\tau_{a}^{\prime}, \mu\right) F\left(\tau_{a}^{\prime}, \mu\right),
$$

and satisfies the RGE

$$
\mu \frac{d}{d \mu} F\left(\tau_{a}, \mu\right)=\int d \tau_{a}^{\prime} \gamma_{F}\left(\tau_{a}-\tau_{a}^{\prime}, \mu\right) F\left(\tau_{a}^{\prime}, \mu\right),
$$

with the anomalous dimension in this case given by

$$
\gamma_{F}\left(\tau_{a}, \mu\right)=-\int d \tau_{a}^{\prime} Z_{F}^{-1}\left(\tau_{a}-\tau_{a}^{\prime}, \mu\right) \mu \frac{d}{d \mu} Z_{F}\left(\tau_{a}^{\prime}, \mu\right),
$$

and taking the general form

$$
\gamma_{F}\left(\tau_{a}, \mu\right)=-\Gamma_{F}\left[\alpha_{s}\right]\left(\frac{2}{j_{F}}\left[\frac{\Theta\left(\tau_{a}\right)}{\tau_{a}}\right]_{+}-\ln \frac{\mu^{2}}{m_{F}^{2}} \delta\left(\tau_{a}\right)\right)+\gamma_{F}\left[\alpha_{s}\right] \delta\left(\tau_{a}\right) .
$$

The solution of eq. (B.10) is

$$
F\left(\tau_{a}, \mu\right)=\int d \tau^{\prime} U_{F}\left(\tau_{a}-\tau_{a}^{\prime}, \mu, \mu_{0}\right) F\left(\tau_{a}^{\prime}, \mu_{0}\right),
$$

where to all orders in $\alpha_{s}$ the evolution kernel $U_{F}$ is given by [74-78]

$$
U_{F}\left(\tau_{a}, \mu, \mu_{0}\right)=\frac{e^{K_{F}+\gamma_{E} \omega_{F}}}{\Gamma\left(-\omega_{F}\right)}\left(\frac{\mu_{0}}{m_{F}}\right)^{j_{F} \omega_{F}}\left[\frac{\Theta\left(\tau_{a}\right)}{\left(\tau_{a}\right)^{1+\omega_{F}}}\right]_{+},
$$

where $\gamma_{E}$ is the Euler constant.

The exponents $\omega_{F}\left(\mu, \mu_{0}\right)$ and $K_{F}\left(\mu, \mu_{0}\right)$ of eqs. (B.8) and (B.14) are given by (where we set $j_{F}=1$ in the multiplicative case of eq. (B.4))

$$
\begin{aligned}
\omega_{F}\left(\mu, \mu_{0}\right) & \equiv \frac{2}{j_{F}} \int_{\alpha_{s}\left(\mu_{0}\right)}^{\alpha_{s}(\mu)} \frac{d \alpha}{\beta[\alpha]} \Gamma_{F}[\alpha] \\
K_{F}\left(\mu, \mu_{0}\right) & \equiv \int_{\alpha_{s}\left(\mu_{0}\right)}^{\alpha_{s}(\mu)} \frac{d \alpha}{\beta[\alpha]} \gamma_{F}[\alpha]+2 \int_{\alpha_{s}\left(\mu_{0}\right)}^{\alpha_{s}(\mu)} \frac{d \alpha}{\beta[\alpha]} \Gamma_{F}[\alpha] \int_{\alpha_{s}\left(\mu_{0}\right)}^{\alpha} \frac{d \alpha^{\prime}}{\beta\left[\alpha^{\prime}\right]} .
\end{aligned}
$$


At NLL (and NLL') accuracy we can write $\omega_{F}\left(\mu, \mu_{0}\right)$ and $K_{F}\left(\mu, \mu_{0}\right)$ as

$$
\begin{aligned}
\left.\omega_{F}\left(\mu, \mu_{0}\right)\right|_{\mathrm{NLL}}= & -\frac{\Gamma_{F}^{0}}{j_{F} \beta_{0}}\left[\ln r+\left(\frac{\Gamma_{c}^{1}}{\Gamma_{c}^{0}}-\frac{\beta_{1}}{\beta_{0}}\right) \frac{\alpha_{s}\left(\mu_{0}\right)}{4 \pi}(r-1)\right], \\
\left.K_{F}\left(\mu, \mu_{0}\right)\right|_{\mathrm{NLL}}=-\frac{\gamma_{F}^{0}}{2 \beta_{0}} \ln r-\frac{2 \pi \Gamma_{F}^{0}}{\left(\beta_{0}\right)^{2}} & {\left[\frac{r-1-r \ln r}{\alpha_{s}(\mu)}\right.} \\
& \left.+\left(\frac{\Gamma_{c}^{1}}{\Gamma_{c}^{0}}-\frac{\beta_{1}}{\beta_{0}}\right) \frac{1-r+\ln r}{4 \pi}+\frac{\beta_{1}}{8 \pi \beta_{0}} \ln ^{2} r\right],
\end{aligned}
$$

where $r=\alpha_{s}(\mu) / \alpha_{s}\left(\mu_{0}\right)$, which can be evaluated at two loops via the equation,

$$
\frac{1}{\alpha_{s}(\mu)}=\frac{1}{\alpha_{s}\left(M_{Z}\right)}+\frac{\beta_{0}}{2 \pi} \ln \left(\frac{\mu}{M_{Z}}\right)+\frac{\beta_{1}}{4 \pi \beta_{0}} \ln \left[1+\frac{\beta_{0}}{2 \pi} \alpha_{s}\left(M_{Z}\right) \ln \left(\frac{\mu}{M_{Z}}\right)\right],
$$

with $\beta_{0}, \beta_{1}$ are the one-loop and two-loop coefficients of the beta function,

$$
\beta\left[\alpha_{s}\right]=\mu \frac{d \alpha_{s}}{d \mu}=-2 \alpha_{s}\left[\beta_{0}\left(\frac{\alpha_{s}}{4 \pi}\right)+\beta_{1}\left(\frac{\alpha_{s}}{4 \pi}\right)^{2}+\cdots\right],
$$

and where (with $T_{R}$ set to $1 / 2$ )

$$
\beta_{0}=\frac{11 C_{A}}{3}-\frac{2 N_{f}}{3} \quad \text { and } \quad \beta_{1}=\frac{34 C_{A}^{2}}{3}-\frac{10 C_{A} N_{f}}{3}-2 C_{F} N_{f} .
$$

In eq. (B.16), we have used that $\Gamma_{F}\left[\alpha_{s}\right]$ for $F=H, J, S$ (hard, jet, and soft) is proportional to $\Gamma_{\text {cusp }}\left[\alpha_{s}\right]$, where

$$
\Gamma_{\text {cusp }}\left[\alpha_{s}\right]=\left(\frac{\alpha_{s}}{4 \pi}\right) \Gamma_{c}^{0}+\left(\frac{\alpha_{s}}{4 \pi}\right)^{2} \Gamma_{c}^{1}+\cdots
$$

Here $\Gamma_{c}^{0}=4$ and the ratio of the one-loop and two-loop coefficients of $\Gamma_{\text {cusp }}$ is [79]

$$
\frac{\Gamma_{c}^{1}}{\Gamma_{c}^{0}}=\left(\frac{67}{9}-\frac{\pi^{2}}{3}\right) C_{A}-\frac{10 N_{f}}{9} .
$$

At NLL', we will need both $\Gamma_{c}^{1}$ and $\beta_{1}$ in the expressions of $\omega_{F}$ and $K_{F}$ for NLL' resummation.

Open Access. This article is distributed under the terms of the Creative Commons Attribution License (CC-BY 4.0), which permits any use, distribution and reproduction in any medium, provided the original author(s) and source are credited.

\section{References}

[1] J.C. Collins, D.E. Soper and G.F. Sterman, Factorization of hard processes in QCD, Adv. Ser. Direct. High Energy Phys. 5 (1989) 1 [hep-ph/0409313] [INSPIRE].

[2] G.F. Sterman, Partons, factorization and resummation, TASI 95, hep-ph/9606312 [INSPIRE]. 
[3] C.W. Bauer, S. Fleming and M.E. Luke, Summing Sudakov logarithms in $\vec{B} X_{s} \gamma$ in effective field theory, Phys. Rev. D 63 (2000) 014006 [hep-ph/0005275] [INSPIRE].

[4] C.W. Bauer, S. Fleming, D. Pirjol and I.W. Stewart, An effective field theory for collinear and soft gluons: heavy to light decays, Phys. Rev. D 63 (2001) 114020 [hep-ph/0011336] [INSPIRE].

[5] C.W. Bauer and I.W. Stewart, Invariant operators in collinear effective theory, Phys. Lett. B 516 (2001) 134 [hep-ph/0107001] [INSPIRE].

[6] C.W. Bauer, D. Pirjol and I.W. Stewart, Soft collinear factorization in effective field theory, Phys. Rev. D 65 (2002) 054022 [hep-ph/0109045] [InSPIRE].

[7] C.W. Bauer, A.V. Manohar and M.B. Wise, Enhanced nonperturbative effects in jet distributions, Phys. Rev. Lett. 91 (2003) 122001 [hep-ph/0212255] [INSPIRE].

[8] C.W. Bauer, C. Lee, A.V. Manohar and M.B. Wise, Enhanced nonperturbative effects in $Z$ decays to hadrons, Phys. Rev. D 70 (2004) 034014 [hep-ph/0309278] [INSPIRE].

[9] S. Fleming, A.H. Hoang, S. Mantry and I.W. Stewart, Jets from massive unstable particles: top-mass determination, Phys. Rev. D 77 (2008) 074010 [hep-ph/0703207] [INSPIRE].

[10] C.W. Bauer, S.P. Fleming, C. Lee and G.F. Sterman, Factorization of $e^{+} e^{-}$event shape distributions with hadronic final states in soft collinear effective theory, Phys. Rev. D 78 (2008) 034027 [arXiv:0801.4569] [inSPIRE].

[11] A.V. Manohar and I.W. Stewart, The zero-bin and mode factorization in quantum field theory, Phys. Rev. D 76 (2007) 074002 [hep-ph/0605001] [INSPIRE].

[12] S.D. Ellis, C.K. Vermilion, J.R. Walsh, A. Hornig and C. Lee, Jet shapes and jet algorithms in SCET, JHEP 11 (2010) 101 [arXiv:1001.0014] [INSPIRE].

[13] Y.-T. Chien, R. Kelley, M.D. Schwartz and H.X. Zhu, Resummation of jet mass at hadron colliders, Phys. Rev. D 87 (2013) 014010 [arXiv: 1208.0010] [INSPIRE].

[14] M. Dasgupta, A. Fregoso, S. Marzani and G.P. Salam, Towards an understanding of jet substructure, JHEP 09 (2013) 029 [arXiv:1307.0007] [INSPIRE].

[15] T.T. Jouttenus, I.W. Stewart, F.J. Tackmann and W.J. Waalewijn, Jet mass spectra in Higgs boson plus one jet at next-to-next-to-leading logarithmic order, Phys. Rev. D 88 (2013) 054031 [arXiv: 1302.0846] [INSPIRE].

[16] A.J. Larkoski, S. Marzani, G. Soyez and J. Thaler, Soft drop, JHEP 05 (2014) 146 [arXiv: 1402.2657] [INSPIRE].

[17] A.J. Larkoski, D. Neill and J. Thaler, Jet shapes with the broadening axis, JHEP 04 (2014) 017 [arXiv: 1401.2158] [inSPIRE].

[18] A.J. Larkoski and J. Thaler, Unsafe but calculable: ratios of angularities in perturbative QCD, JHEP 09 (2013) 137 [arXiv:1307.1699] [INSPIRE].

[19] A.J. Larkoski, G.P. Salam and J. Thaler, Energy correlation functions for jet substructure, JHEP 06 (2013) 108 [arXiv: 1305.0007] [INSPIRE].

[20] M. Dasgupta, K. Khelifa-Kerfa, S. Marzani and M. Spannowsky, On jet mass distributions in $Z+$ jet and dijet processes at the LHC, JHEP 10 (2012) 126 [arXiv:1207.1640] [INSPIRE].

[21] M. Dasgupta, A. Fregoso, S. Marzani and A. Powling, Jet substructure with analytical methods, Eur. Phys. J. C 73 (2013) 2623 [arXiv:1307.0013] [InSPIRE].

[22] T. Becher and X. Garcia i Tormo, Factorization and resummation for transverse thrust, JHEP 06 (2015) 071 [arXiv: 1502.04136] [INSPIRE]. 
[23] Y.-T. Chien and I. Vitev, Jet shape resummation using soft-collinear effective theory, JHEP 12 (2014) 061 [arXiv: 1405.4293] [INSPIRE].

[24] A.J. Larkoski, I. Moult and D. Neill, Analytic boosted boson discrimination, arXiv: 1507.03018 [INSPIRE].

[25] S. Fleming, A.K. Leibovich and T. Mehen, Resummation of large endpoint corrections to color-octet $J / \psi$ photoproduction, Phys. Rev. D 74 (2006) 114004 [hep-ph/0607121] [INSPIRE].

[26] I.W. Stewart, F.J. Tackmann and W.J. Waalewijn, Factorization at the LHC: from PDFs to initial state jets, Phys. Rev. D 81 (2010) 094035 [arXiv:0910.0467] [INSPIRE].

[27] S. Alioli and J.R. Walsh, Jet veto clustering logarithms beyond leading order, JHEP 03 (2014) 119 [arXiv:1311.5234] [INSPIRE].

[28] R. Kelley, J.R. Walsh and S. Zuberi, Disentangling clustering effects in jet algorithms, arXiv:1203.2923 [INSPIRE].

[29] R. Kelley, J.R. Walsh and S. Zuberi, Abelian non-global logarithms from soft gluon clustering, JHEP 09 (2012) 117 [arXiv:1202.2361] [INSPIRE].

[30] M. Dasgupta and G.P. Salam, Resummation of nonglobal QCD observables, Phys. Lett. B 512 (2001) 323 [hep-ph/0104277] [INSPIRE].

[31] A. Banfi, G. Marchesini and G. Smye, Away from jet energy flow, JHEP 08 (2002) 006 [hep-ph/0206076] [INSPIRE].

[32] R.B. Appleby and G.P. Salam, Theory and phenomenology of nonglobal logarithms, hep-ph/0305232 [INSPIRE].

[33] Y.L. Dokshitzer and G. Marchesini, On large angle multiple gluon radiation, JHEP 03 (2003) 040 [hep-ph/0303101] [INSPIRE].

[34] M. Dasgupta, F. Dreyer, G.P. Salam and G. Soyez, Small-radius jets to all orders in QCD, JHEP 04 (2015) 039 [arXiv:1411.5182] [INSPIRE].

[35] T. Becher, M. Neubert, L. Rothen and D.Y. Shao, An effective field theory for jet processes, arXiv:1508.06645 [INSPIRE].

[36] Y.-T. Chien, A. Hornig and C. Lee, Soft-collinear mode for jet cross sections in soft collinear effective theory, Phys. Rev. D 93 (2016) 014033 [arXiv: 1509.04287] [INSPIRE].

[37] K. Khelifa-Kerfa and Y. Delenda, Non-global logarithms at finite $N_{c}$ beyond leading order, JHEP 03 (2015) 094 [arXiv: 1501.00475] [INSPIRE].

[38] A. Hornig, C. Lee, J.R. Walsh and S. Zuberi, Double non-global logarithms in-n-out of jets, JHEP 01 (2012) 149 [arXiv:1110.0004] [INSPIRE].

[39] A. Hornig, C. Lee, I.W. Stewart, J.R. Walsh and S. Zuberi, Non-global structure of the $O\left(\alpha_{s}^{2}\right)$ dijet soft function, JHEP 08 (2011) 054 [arXiv:1105.4628] [INSPIRE].

[40] R. Kelley, M.D. Schwartz, R.M. Schabinger and H.X. Zhu, Jet mass with a jet veto at two loops and the universality of non-global structure, Phys. Rev. D 86 (2012) 054017 [arXiv:1112.3343] [INSPIRE].

[41] A.J. Larkoski, I. Moult and D. Neill, Non-global logarithms, factorization and the soft substructure of jets, JHEP 09 (2015) 143 [arXiv:1501.04596] [INSPIRE].

[42] D. Neill, The edge of jets and subleading non-global logs, arXiv:1508.07568 [INSPIRE]. 
[43] L.G. Almeida et al., Substructure of high-p $p_{T}$ jets at the LHC, Phys. Rev. D 79 (2009) 074017 [arXiv: 0807.0234] [INSPIRE].

[44] C.F. Berger, T. Kucs and G.F. Sterman, Event shape/energy flow correlations, Phys. Rev. D 68 (2003) 014012 [hep-ph/0303051] [INSPIRE].

[45] C. Lee and G.F. Sterman, Universality of nonperturbative effects in event shapes, eConf C 0601121 (2006) A001 [hep-ph/0603066] [INSPIRE].

[46] A. Hornig, C. Lee and G. Ovanesyan, Effective predictions of event shapes: factorized, resummed and gapped angularity distributions, JHEP 05 (2009) 122 [arXiv:0901.3780] [INSPIRE].

[47] S.D. Ellis, Z. Kunszt and D.E. Soper, Jets at hadron colliders at order $\alpha_{s}^{3}$ : a look inside, Phys. Rev. Lett. 69 (1992) 3615 [hep-ph/9208249] [INSPIRE].

[48] S.D. Ellis, Z. Kunszt and D.E. Soper, Jets in hadron colliders at order $\alpha_{s}^{3}$, Conf. Proc. C 910725V1 (1991) 417 [INSPIRE].

[49] Y.-T. Chien and I. Vitev, Towards the understanding of jet shapes and cross sections in heavy ion collisions using soft-collinear effective theory, arXiv:1509.07257 [INSPIRE].

[50] I.W. Stewart, F.J. Tackmann and W.J. Waalewijn, $N$ jettiness: an inclusive event shape to veto jets, Phys. Rev. Lett. 105 (2010) 092002 [arXiv:1004.2489] [INSPIRE].

[51] Z.L. Liu, C.S. Li, J. Wang and Y. Wang, Resummation prediction on the jet mass spectrum in one-jet inclusive production at the LHC, JHEP 04 (2015) 005 [arXiv:1412.1337] [INSPIRE].

[52] L.G. Almeida et al., Comparing and counting logs in direct and effective methods of QCD resummation, JHEP 04 (2014) 174 [arXiv:1401.4460] [INSPIRE].

[53] C.W. Bauer, N.D. Dunn and A. Hornig, Subtractions for SCET soft functions, arXiv:1102. 4899 [INSPIRE].

[54] G.P. Salam, Towards jetography, Eur. Phys. J. C 67 (2010) 637 [arXiv:0906.1833] [INSPIRE].

[55] M. Procura and W.J. Waalewijn, Fragmentation in jets: cone and threshold effects, Phys. Rev. D 85 (2012) 114041 [arXiv:1111.6605] [InSPIRE].

[56] M. Ritzmann and W.J. Waalewijn, Fragmentation in jets at NNLO, Phys. Rev. D 90 (2014) 054029 [arXiv: 1407.3272] [INSPIRE].

[57] M. Procura and I.W. Stewart, Quark fragmentation within an identified jet, Phys. Rev. D 81 (2010) 074009 [Erratum ibid. D 83 (2011) 039902] [arXiv:0911.4980] [INSPIRE].

[58] R.K. Ellis, W.J. Stirling and B.R. Webber, $Q C D$ and collider physics, Camb. Monogr. Part. Phys. Nucl. Phys. Cosmol. 8 (1996) 1 [inSPIRE].

[59] S. Catani and M.H. Seymour, The dipole formalism for the calculation of QCD jet cross-sections at next-to-leading order, Phys. Lett. B 378 (1996) 287 [hep-ph/9602277] [INSPIRE].

[60] S. Catani and M.H. Seymour, A general algorithm for calculating jet cross-sections in NLO QCD, Nucl. Phys. B 485 (1997) 291 [Erratum ibid. B 510 (1998) 503] [hep-ph/9605323] [INSPIRE].

[61] J.-y. Chiu, A. Jain, D. Neill and I.Z. Rothstein, The rapidity renormalization group, Phys. Rev. Lett. 108 (2012) 151601 [arXiv:1104.0881] [INSPIRE]. 
[62] J.-y. Chiu, A. Fuhrer, R. Kelley and A.V. Manohar, Factorization structure of gauge theory amplitudes and application to hard scattering processes at the LHC,

Phys. Rev. D 80 (2009) 094013 [arXiv: 0909.0012] [INSPIRE].

[63] T. Becher and M. Neubert, On the structure of infrared singularities of gauge-theory amplitudes, JHEP 06 (2009) 081 [Erratum ibid. 11 (2013) 024] [arXiv:0903.1126] [INSPIRE].

[64] R. Kelley and M.D. Schwartz, 1-loop matching and NNLL resummation for all partonic 2 to 2 processes in QCD, Phys. Rev. D 83 (2011) 045022 [arXiv: 1008.2759] [INSPIRE].

[65] Z. Ligeti, I.W. Stewart and F.J. Tackmann, Treating the $b$ quark distribution function with reliable uncertainties, Phys. Rev. D 78 (2008) 114014 [arXiv:0807.1926] [INSPIRE].

[66] R. Abbate, M. Fickinger, A.H. Hoang, V. Mateu and I.W. Stewart, Thrust at $N^{3} L L$ with power corrections and a precision global fit for $\alpha_{s}\left(m_{Z}\right)$, Phys. Rev. D 83 (2011) 074021 [arXiv: 1006.3080] [INSPIRE].

[67] T. Becher, G. Bell and M. Neubert, Factorization and resummation for jet broadening, Phys. Lett. B 704 (2011) 276 [arXiv:1104.4108] [InSPIRE].

[68] J.-Y. Chiu, A. Jain, D. Neill and I.Z. Rothstein, A formalism for the systematic treatment of rapidity logarithms in quantum field theory, JHEP 05 (2012) 084 [arXiv:1202.0814] [INSPIRE].

[69] I.W. Stewart, F.J. Tackmann and W.J. Waalewijn, The beam thrust cross section for Drell-Yan at NNLL order, Phys. Rev. Lett. 106 (2011) 032001 [arXiv:1005.4060] [INSPIRE].

[70] R. Bain et al., Fragmenting jet functions with angularities, in preparation.

[71] X. Liu, SCET approach to top quark decay, Phys. Lett. B 699 (2011) 87 [arXiv:1011.3872] [INSPIRE].

[72] A. Jain, M. Procura and W.J. Waalewijn, Parton fragmentation within an identified jet at NNLL, JHEP 05 (2011) 035 [arXiv:1101.4953] [INSPIRE].

[73] A. Jain, M. Procura and W.J. Waalewijn, Fully-unintegrated parton distribution and fragmentation functions at perturbative $k_{T}$, JHEP 04 (2012) 132 [arXiv:1110.0839] [INSPIRE].

[74] G.P. Korchemsky and G. Marchesini, Resummation of large infrared corrections using Wilson loops, Phys. Lett. B 313 (1993) 433 [INSPIRE].

[75] T. Becher, M. Neubert and B.D. Pecjak, Factorization and momentum-space resummation in deep-inelastic scattering, JHEP 01 (2007) 076 [hep-ph/0607228] [INSPIRE].

[76] C. Balzereit, T. Mannel and W. Kilian, Evolution of the light cone distribution function for a heavy quark, Phys. Rev. D 58 (1998) 114029 [hep-ph/9805297] [INSPIRE].

[77] M. Neubert, Advanced predictions for moments of the $\bar{B} \rightarrow X_{s} \gamma$ photon spectrum, Phys. Rev. D 72 (2005) 074025 [hep-ph/0506245] [INSPIRE].

[78] S. Fleming, A.H. Hoang, S. Mantry and I.W. Stewart, Top jets in the peak region: factorization analysis with next-to-leading-log resummation, Phys. Rev. D 77 (2008) 114003 [arXiv: 0711.2079] [INSPIRE].

[79] G.P. Korchemsky and A.V. Radyushkin, Renormalization of the Wilson loops beyond the leading order, Nucl. Phys. B 283 (1987) 342 [inSPIRE]. 